\title{
Three-Dimensional Investigation of the Stokes Eigenmodes in Hollow Circular Cylinder
}

\author{
Adil El Baroudi ${ }^{1}$ and Fulgence Razafimahery ${ }^{2}$ \\ ${ }^{1}$ Arts et Métiers ParisTech, ENSAM Angers, 2 Boulevard du Ronceray, 49035 Angers, France \\ ${ }^{2}$ IRMAR, Université de Rennes 1, Campus de Beaulieu, 35042 Rennes Cedex, France \\ Correspondence should be addressed to Adil El Baroudi; adil.elbaroudi@ensam.eu
}

Received 8 July 2013; Revised 29 August 2013; Accepted 30 August 2013

Academic Editor: K. M. Liew

Copyright (C) 2013 A. El Baroudi and F. Razafimahery. This is an open access article distributed under the Creative Commons Attribution License, which permits unrestricted use, distribution, and reproduction in any medium, provided the original work is properly cited.

\begin{abstract}
This paper studies the influence of boundary conditions on a fluid medium of finite depth. We determine the frequencies and the modal shapes of the fluid. The fluid is assumed to be incompressible and viscous. A potential technique is used to obtain in threedimensional cylindrical coordinates a general solution for a problem. The method consists in solving analytically partial differential equations obtained from the linearized Navier-Stokes equation. A finite element analysis is also used to check the validity of the present method. The results from the proposed method are in good agreement with numerical solutions. The effect of the fluid thickness on the Stokes eigenmodes is also investigated. It is found that frequencies are strongly influenced.
\end{abstract}

\section{Introduction}

Flow modeling in confined domains leads mostly to Stokes models [1]. The use of this model in the field of microfluidics and nanofluidics $[2,3]$ and in the understanding the movement of microorganisms [4] currently in full swing. This recent years, several authors have focused on the modal analysis of this model. We can cite in particular the aspects related to problems arising from geophysics. These studies highlight the existence of slow wave or Stoneley waves [5-7]. Other authors $[8,9]$ have focused on the dynamic aspects in the context of fluid-structure interaction. The numeric aspects for coupled (or not) modal problem were discussed in [10-12].

The theory of potential flow of viscous fluid was introduced by [13]. All of his work on this topic is framed in terms of the effects of viscosity on the attenuation of small amplitude waves on a liquid-gas surface. The problem treated by Stokes was solved exactly using the linearized Navier-Stokes equations, without assuming potential flow, and was solved exactly by [14]. Reference [15] has identified the main events in the history of thought about potential flow of viscous fluids. The problem of Stokes flow in cylindrical domain has been investigated by several authors. References $[16,17]$ studied
Stokes flow in a cylindrical container by an eigenfunction expansion procedure without the compressibility effect.

Potential flows through different kinds of geometry have been studied by many investigators for several applications. For example in vascular fluid dynamics, [18] presented the role of curvature in the wave propagation and in the development of a secondary flow. Reference [19] has studied the flow and pressure dynamics of the cerebrospinal fluid flow. Threedimensional CSF flow studies have also been reported [20].

The knowledge of Stokes eigenmodes in a three-dimensional confined domain, in a square/cube [10], or in any bounded domain could provide some insight into the understanding or analysis of turbulent instantaneous flow field in a geometry as simple as, for instance, the driven cavity.

This paper deals with analytical and numerical modeling of three-dimensional incompressible viscous fluid using the potential technique and the finite element method. The analytical formulation is based upon a convenient decomposition of the velocity field into two contributions [9-21]: one is related to the scalar potential and the other is the vector potential.

The rest of the paper is organized as follows. In Section 2, we write the linearized Navier-Stokes equation, a simple 
representation for the velocity field in terms of the potential. The implementation of analytical solutions is discussed in detail. Section 3 describes boundary condition treatment for modeling Stokes eigenmodes. Section 4 investigates the case of fluid-solid interaction. Section 5 is completely devoted to the analytical and numerical results. Finally, the paper is ended by some conclusions.

\section{Governing Equations}

From conservations of mass and momentum and assuming that the fluid is viscous and incompressible, the motion of the fluid flowing in the cylindrical waveguide in the absence of body force is governed by

$$
\begin{gathered}
\rho \frac{\partial \mathbf{v}(r, \theta, z)}{\partial t}=-\nabla p(r, \theta, z)+\eta \nabla^{2} \mathbf{v}(r, \theta, z), \\
\nabla \cdot \mathbf{v}(r, \theta, z)=0,
\end{gathered}
$$

in which $\mathbf{v}(r, \theta, z)=\left\{v_{r}, v_{\theta}, v_{z}\right\}^{T}(r, \theta, z)$ is the fluid velocity vector, $p(r, \theta, z)$ is the fluid pressure, $\rho$ is the density of the fluid, and $v$ and $\eta=\rho \nu$ are the kinematic and dynamic fluid viscosity, respectively.

The obtained equations of motion are highly complex and coupled. However, a simpler set of equations can be obtained by introducing scalar potentials $\phi, \psi$, and $\chi$, known as the Helmholtz decomposition. The Helmholtz decomposition theorem [22] states that any vector $\mathbf{v}$ can be written as the sum of two parts: one is curl-free and the other is solenoidal. In flow fields, the velocity is thereby decomposed into a potential flow and a viscous flow. In other words, the velocity $\mathbf{v}$ can be decomposed into the following form:

$$
\mathbf{v}=\nabla \phi+\nabla \times\left(\psi \mathbf{e}_{\mathbf{z}}\right)+\nabla \times \nabla \times\left(\chi \mathbf{e}_{\mathbf{z}}\right),
$$

where $\mathbf{e}_{\mathbf{z}}$ is the unit vector along the $z$ direction. Substituting the above resolutions into (1) and (2), after some manipulations, the equation for the conservation of mass (2) becomes the Laplace equation:

$$
\nabla^{2} \phi(r, \theta, z)=0
$$

and the equation for the conservation of momentum (1) becomes the Helmholtz equations

$$
\begin{aligned}
& \nabla^{2} \psi(r, \theta, z)-\frac{1}{v} \frac{\partial \psi(r, \theta, z)}{\partial t}=0, \\
& \nabla^{2} \chi(r, \theta, z)-\frac{1}{v} \frac{\partial \chi(r, \theta, z)}{\partial t}=0,
\end{aligned}
$$

where $\nabla^{2}=\left(\partial^{2} / \partial r^{2}\right)+(1 / r)(\partial / \partial r)+\left(1 / r^{2}\right)\left(\partial^{2} / \partial \theta^{2}\right)+\left(\partial^{2} /\right.$ $\left.\partial z^{2}\right)$ is the Laplacian operator in polar coordinates, and time dependence has the form $\exp (j \omega t)$. The pressure can be presented by

$$
p=-\rho \frac{\partial \phi}{\partial t} .
$$

Thus, the Navier-Stokes equations are reduced to formulations (4), (5), and (6). Of course, it does not mean that in any case such a decomposition of fluid's velocity field gives considerable simplifications in solving the problem because boundary conditions for stresses are not separated. The vibrations are harmonic, with constant angular frequency $\omega$; as a result all potentials components and pressure will depend on time only through the factor $\exp (j \omega t)$.

Applying the method of separation of variables, the solution of the equations for potentials, associated with an axial wave number $k_{z}$, radial wave number $k_{\psi}$, and circumferential mode parameter $n$, after considerable algebraic manipulations, can be shown to be

$$
\begin{aligned}
\phi= & {\left[A I_{n}\left(k_{z} r\right)+B K_{n}\left(k_{z} r\right)\right]\left\{\begin{array}{l}
\sin (n \theta) \\
\cos (n \theta)
\end{array}\right\} } \\
& \times\left\{\begin{array}{l}
\cos \left(k_{z} z\right) \\
\sin \left(k_{z} z\right)
\end{array}\right\}, \\
\psi= & {\left[C J_{n}\left(k_{\psi} r\right)+D Y_{n}\left(k_{\psi} r\right)\right]\left\{\begin{array}{l}
\cos (n \theta) \\
\sin (n \theta)
\end{array}\right\} } \\
& \times\left\{\begin{array}{l}
\cos \left(k_{z} z\right) \\
\sin \left(k_{z} z\right)
\end{array}\right\}, \\
\chi= & {\left[E J_{n}\left(k_{\psi} r\right)+F Y_{n}\left(k_{\psi} r\right)\right]\left\{\begin{array}{l}
\sin (n \theta) \\
\cos (n \theta)
\end{array}\right\} } \\
& \times\left\{\begin{array}{l}
\sin \left(k_{z} z\right) \\
\cos \left(k_{z} z\right)
\end{array}\right\} .
\end{aligned}
$$

$J_{n}$ and $Y_{n}$ are Bessel functions of the first and second kind of order $n$. $I_{n}$ and $K_{n}$ are modified Bessel functions of the first and second kinds of order $n . n$ and $k_{z}$ are the azimuthal and axial wavenumbers. $n=0,1,2, \ldots$, whereas $k_{z}$ is found by satisfying the symmetry boundary condition on the $z=0$ and $z=l$. Note that the velocity field $\mathbf{v}$ has components that are symmetric or antisymmetric in $\theta$ and $z$. Following standard practice, the solutions with symmetric (antisymmetric) axial velocities are called the antisymmetric (symmetric) axial modes, respectively, with $k_{z}^{a}$ and $k_{z}^{s}$ denoting the corresponding eigenvalues. Thus, we have $k_{z}^{a}=(m-1) \pi / l$ and $k_{z}^{s}=m \pi / l(m=0,1,2, \ldots)$. However, the azimuthal modes corresponding to $\cos (n \theta)$ and $\sin (n \theta)$ are really the same, due to periodicity in the azimuthal direction; that is, there is no distinction in the values of $n$ for the two families. $A, B, C, D, E$, and $F$ are unknown coefficients which will be determined later by imposing the appropriate boundary conditions. The radial wave number $k_{\psi}$ is related to the axial wave number $k_{z}$ by

$$
k_{\psi}^{2}=\alpha^{2}-k_{z}^{2}, \quad \omega=j v \alpha^{2},
$$

where $j=\sqrt{-1}$. In the end, the pressure can be obtained by insertion of (7) into (6),

$$
p=\eta \alpha^{2}\left[A I_{n}\left(k_{z} r\right)+B K_{n}\left(k_{z} r\right)\right] \cos (n \theta) \cos \left(k_{z} z\right) .
$$


Using (3) and taking into account (7), (8), and (9), the fluid particle velocities in terms of the Bessel functions can be written as

$$
\begin{aligned}
v_{r}=\{ & A I_{n}^{\prime}\left(k_{z} r\right)+B K_{n}^{\prime}\left(k_{z} r\right)-\frac{n}{r} C J_{n}\left(k_{\psi} r\right)-\frac{n}{r} D Y_{n}\left(k_{\psi} r\right) \\
& \left.+k_{z} E J_{n}^{\prime}\left(k_{\psi} r\right)+k_{z} F Y_{n}^{\prime}\left(k_{\psi} r\right)\right\} \\
& \times \sin (n \theta) \cos \left(k_{z} z\right), \\
v_{\theta}= & \left\{\frac{n}{r} A I_{n}\left(k_{z} r\right)+\frac{n}{r} B K_{n}\left(k_{z} r\right)-C J_{n}^{\prime}\left(k_{\psi} r\right)\right. \\
& \left.\quad-D Y_{n}^{\prime}\left(k_{\psi} r\right)+\frac{n k_{z}}{r}\left[E J_{n}\left(k_{\psi} r\right)+F Y_{n}\left(k_{\psi} r\right)\right]\right\} \\
& \times \cos (n \theta) \cos \left(k_{z} z\right), \\
v_{z}= & \left\{k_{\psi}^{2} E J_{n}\left(k_{\psi} r\right)+k_{\psi}^{2} F Y_{n}\left(k_{\psi} r\right)-k_{z} A I_{n}\left(k_{z} r\right)\right. \\
& \left.\quad-k_{z} B K_{n}\left(k_{z} r\right)\right\} \sin (n \theta) \sin \left(k_{z} z\right) .
\end{aligned}
$$

In the following section we consider a fluid with different boundary conditions.

\section{Boundary Conditions and Frequency Equation}

First we define $\Gamma_{1}\left(r=R_{1}\right)$, as the inner boundary of the fluid region, and $\Gamma_{2}\left(r=R_{2}\right)$, as the outer boundary. We begin the analysis of frequency equation for imposing the no-slip boundary conditions.

3.1. Stokes Eigenmodes in the Case of "No-Slip-No-Slip" Boundary Conditions. The no-slip hypothesis of fluid mechanics states that liquid velocity at a solid surface is equal to the velocity of the solid surface. Hence the no-slip boundary condition can be written as

(1) the velocity is equal to zero at $\Gamma_{1}$ :

$$
v_{r}=v_{\theta}=v_{z}=0,
$$

(2) the velocity is equal to zero at $\Gamma_{2}$ :

$$
v_{r}=v_{\theta}=v_{z}=0 \text {. }
$$

Combining these boundary conditions with (12) yields for each mode number $(n, m)$ the following linear system:

$$
\left[\mathbf{M}_{1}\right]\{x\}=\{0\}, \quad\{x\}=\{A B C D E F\}^{T},
$$

where $\left[\mathbf{M}_{1}\right]$ is a $6 \times 6$ matrix whose components $a_{i j}$ are given in Appendix A. For a non-trivial solution, the determinant of the matrix $\left[\mathbf{M}_{1}\right]$ must be equal to zero

$$
\operatorname{det}\left[\mathbf{M}_{1}\right]=0 \text {. }
$$

The roots of (16) give the natural frequencies $\omega$ of the cylindrical oscillations. Figure 16 in Appendix D shows analytical calculation of eigenvalues $\alpha$ and using (10) we obtain the natural frequencies $\omega[\mathrm{rd} / \mathrm{s}]$.
3.2. Stokes Eigenmodes in the Case of "No-Slip-Normal Stress-Free" Boundary Conditions. The pressure and velocity boundary conditions on the free surface are both formulated from the dynamic constraint of continuity of normal momentum flux across the free surface. The component of the stress tensor in the outward normal direction is therefore

$$
\sigma \cdot \mathbf{n}=\left\{-p \mathbf{I}+\eta\left[\nabla \mathbf{v}+(\nabla \mathbf{v})^{T}\right]\right\}, \quad \mathbf{n}=0,
$$

in which $\mathbf{I}$ is a unit tensor. By applying this condition on $\Gamma_{1}$ we obtain

$$
\sigma_{r r}=\sigma_{r \theta}=\sigma_{r z}=0 \text {. }
$$

Combining these boundary conditions and (14) yields for each mode number $(n, m)$ the following linear system:

$$
\left[\mathbf{M}_{2}\right]\{\mathbf{x}\}=\{0\}, \quad\{\mathbf{x}\}=\{A B C D E F\}^{T},
$$

where $\left[\mathbf{M}_{2}\right]$ is a $6 \times 6$ matrix whose components $b_{i j}$ are given in Appendix B. For a nontrivial solution, the determinant of the matrix $\left[\mathbf{M}_{2}\right]$ must be equal to zero

$$
\operatorname{det}\left[\mathbf{M}_{2}\right]=0 \text {. }
$$

The roots of (20) give the natural frequencies $\omega$ of the cylindrical oscillations.

3.3. Torsional, Flexural, and Breathing Stokes Eigenmode. The results presented in (16) and (20) are a general natural frequencies equation. For some simpler modes, the abovementioned method can be simplified. For example, we have the following.

3.3.1. Torsional Stokes Eigenmode. The torsion mode vibration is such a mode in which the scalar components of the velocity $\left\{v_{r}, v_{z}\right\}$ are zeros and only the circumferential velocity $v_{\theta}$ is independent of $\theta$. This condition is achieved if $\phi=0$ and $\chi=0$. Through (3) this gives for the nonvanishing components of displacement and stresses:

$$
v_{\theta}=-\frac{\partial \psi}{\partial r} .
$$
the set

Thus, the general solution for $\psi$ must be constructed from

$$
\psi(r, z)=\left[C J_{0}\left(k_{\psi} r\right)+D Y_{0}\left(k_{\psi} r\right)\right] \sin \left(k_{z} z\right) .
$$

In this case the boundary conditions equations (13) and (14) become

$$
\begin{array}{ll}
v_{\theta}=0 & \text { at } r=R_{1}, \\
v_{\theta}=0 & \text { at } r=R_{2} .
\end{array}
$$

Then, (15) becomes

$$
[\mathbf{T}]\{\mathbf{x}\}=\{\mathbf{0}\}, \quad\{\mathbf{x}\}=\{C D\}^{T} .
$$

[T] is a $2 \times 2$ matrix whose components are calculated using Appendices $\mathrm{A}$ and $\mathrm{B}$. Solving $\operatorname{det}[\mathbf{T}]=0$ gives the torsional modes. 
3.4. Longitudinal Stokes Eigenmode. Another simpler mode vibration is called longitudinal mode vibration in which $v_{\theta}=$ 0 and $\left\{v_{r}, v_{z}\right\}$ are independent of $\theta$. This means that the motion is confined to planes perpendicular to the $z$-axis, which can move, expand, and contract in their planes. The solution for the displacement field and stress vector follows from (3) and (17):

$$
\begin{gathered}
v_{r}=\frac{\partial \phi}{\partial r}+k_{z} \frac{\partial \chi}{\partial r} \\
v_{z}=-k_{z} \phi+k_{\psi}^{2} \chi \\
\sigma_{r r}=2 \eta\left\{\frac{\partial^{2} \phi}{\partial r^{2}}-\frac{\alpha^{2}}{2} \phi+k_{z} \frac{\partial^{2} \chi}{\partial r^{2}}\right\}, \\
\sigma_{r z}=\eta\left\{-2 k_{z} \frac{\partial \phi}{\partial r}+\left(k_{\psi}^{2}-k_{z}^{2}\right) \frac{\partial \chi}{\partial r}\right\} .
\end{gathered}
$$

Thus, the general solution for $\phi$ and $\chi$ must be constructed from the set:

$$
\begin{aligned}
& \phi=\left[A J_{0}\left(k_{z} r\right)+B Y_{0}\left(k_{z} r\right)\right] \sin \left(k_{z} z\right), \\
& \chi=\left[E J_{0}\left(k_{\psi} r\right)+F Y_{0}\left(k_{\psi} r\right)\right] \cos \left(k_{z} z\right) .
\end{aligned}
$$

In this case the boundary conditions equations (14) and (18) become

$$
\begin{array}{cc}
\sigma_{r r}=\sigma_{r z}=0 & \text { at } r=R_{1}, \\
v_{r}=v_{z}=0 & \text { at } r=R_{2} .
\end{array}
$$

Then, (19) becomes

$$
[\mathbf{L}]\{\mathbf{x}\}=\{0\}, \quad\{\mathbf{x}\}=\{A B E F\}^{T} .
$$

[L] is a $4 \times 4$ matrix whose components are calculated using the Appendices $\mathrm{A}$ and $\mathrm{B}$. Solving $\operatorname{det}[\mathbf{L}]=0$ gives the longitudinal modes.

3.4.1. Flexural and Breathing Stokes Eigenmodes. The mode shape $n=1$ is called flexural mode vibration in which all components of the displacement are nonvanishing and depend on $r, \theta$, and $z$. The mode shape $n \geqslant 2$ is called breathing mode vibration in which all components of the displacement are non-vanishing and depend on $r, \theta$, and $z$.

In the following we will introduce the interest of the fluid-structure interaction and we will study the influence of viscosity on the eigenmodes of an elastic solid. For this, the inner boundary of the fluid region $\Gamma_{1}$ is represented by an elastic wall.

\section{Fluid-Structure Interaction}

Fluid-structure interaction problems have long since attracted the attention of engineers and applied mathematics. The most important applications of this theory, are probably, structural acoustics [23], vibrations of fluid-conveying pipes $[24,25]$, and biomechanics. As these problems are rather complicated, some simplifications are typically adopted to facilitate their solving. In particular, it is quite typical to ignore viscosity effects (especially in structural acoustics) or to use local theories of interaction, such as the one referred to as thin layer or plane wave approximation.

4.1. Governing Equations of Elastic Media. The wave motion in an isotropic elastic medium is governed by the classical Navier's equation:

$$
-\rho_{s} \omega^{2} \mathbf{u}=\mu \nabla^{2} \mathbf{u}+(\lambda+\mu) \nabla \nabla \cdot \mathbf{u},
$$

where $\rho_{s}$ is the density, $\lambda, \mu$ are the Lamé constants, and $\mathbf{u}(r, \theta, z)=\left\{u_{r}, u_{\theta}, u_{z}\right\}^{T}(r, \theta, z)$ is the vector displacement of particles.

The obtained equations of motion are highly complex and coupled. However, a simpler set of equations can be obtained by introducing scalar potentials $\Phi, \Psi$, and $\Theta$, known as the Helmholtz decomposition such that

$$
\mathbf{u}=\nabla \Phi+\nabla \times\left(\Psi \mathbf{e}_{\mathbf{z}}\right)+\nabla \times \nabla \times\left(\Theta \mathbf{e}_{\mathbf{z}}\right)
$$

Substituting (30) into (29) leads to three sets of differential equations

$$
\begin{aligned}
& \nabla^{2} \Phi(r, \theta, z)-\frac{\nu^{2} \alpha^{4}}{c_{L}^{2}} \Phi(r, \theta, z)=0, \\
& \nabla^{2} \Psi(r, \theta, z)-\frac{\nu^{2} \alpha^{4}}{c_{T}^{2}} \Psi(r, \theta, z)=0, \\
& \nabla^{2} \Theta(r, \theta, z)-\frac{\nu^{2} \alpha^{4}}{c_{T}^{2}} \Theta(r, \theta, z)=0,
\end{aligned}
$$

where $c_{L}=\sqrt{(\lambda+2 \mu) / \rho_{s}}$ and $c_{T}=\sqrt{\mu / \rho_{s}}$ are the compressional and shear wave velocities in the solids, respectively. Applying the method of separation of variables, the solution of the equations for potentials, associated with an axial wave number $k_{z}$, radial wave number $\left(k_{\Phi}, k_{\Psi}\right)$, and circumferential mode parameter $n$, after considerable algebraic manipulations, can be shown to be

$$
\begin{aligned}
& \Phi=\left[a I_{n}\left(k_{\Phi} r\right)+b K_{n}\left(k_{\Phi} r\right)\right] \sin (n \theta) \cos \left(k_{z} z\right), \\
& \Psi=\left[c I_{n}\left(k_{\Psi} r\right)+d K_{n}\left(k_{\Psi} r\right)\right] \cos (n \theta) \cos \left(k_{z} z\right), \\
& \Theta=\left[e I_{n}\left(k_{\Psi} r\right)+f K_{n}\left(k_{\Psi} r\right)\right] \sin (n \theta) \sin \left(k_{z} z\right) .
\end{aligned}
$$

The radial wave number $\left(k_{\Phi}, k_{\Psi}\right)$ is related to the axial wave number $k_{z}$ by

$$
k_{\Phi}^{2}=\frac{v^{2} \alpha^{4}}{c_{L}^{2}}+k_{z}^{2}, \quad k_{\Psi}^{2}=\frac{v^{2} \alpha^{4}}{c_{T}^{2}}+k_{z}^{2}
$$

and $a, b, c, d, e$, and $f$ are unknown coefficients which will be determined later by imposing the appropriate boundary conditions. 
Using (30) the scalar components of the displacement vector $\mathbf{u}$ in cylindrical coordinates can be expressed by

$$
\begin{gathered}
u_{r}=\frac{\partial \Phi}{\partial r}-\frac{n}{r} \Psi+k_{z} \frac{\partial \Theta}{\partial r} \\
u_{\theta}=\frac{n}{r} \Phi-\frac{\partial \Psi}{\partial r}+\frac{n k_{z}}{r} \Theta \\
u_{z}=-k_{z} \Phi-k_{\Psi}^{2} \Theta
\end{gathered}
$$

and the radial and tangential stresses are given by Hooke's law as

$$
\begin{gathered}
\Sigma_{r r}=\lambda \frac{v^{2} \alpha^{4}}{c_{L}^{2}} \Phi+2 \mu \frac{\partial u_{r}}{\partial r}, \\
\Sigma_{r \theta}=\mu\left\{\frac{\partial u_{\theta}}{\partial r}-\frac{u_{\theta}}{r}+\frac{1}{r} \frac{\partial u_{r}}{\partial \theta}\right\}, \\
\Sigma_{r z}=\mu\left\{\frac{\partial u_{r}}{\partial z}+\frac{\partial u_{z}}{\partial r}\right\} .
\end{gathered}
$$

4.2. Boundary Condition and Frequency Equation. We define $\Gamma_{1}\left(r=R_{1}\right)$ as the boundary contact between the fluid region and the solid region and $\Gamma_{2}\left(r=R_{2}\right)$ as the outer boundary. The relevant boundary conditions can be taken as follows.

(1) The normal components of the solid stresses must be zero on the interface $\Gamma_{0}$ :

$$
\Sigma_{r r}=\Sigma_{r \theta}=\Sigma_{r z}=0 .
$$

(2) Kinematic boundary condition (velocity must be continuous) on the interface $\Gamma_{1}$ :

$$
v_{r}=j \omega u_{r}, \quad v_{\theta}=j \omega u_{\theta}, \quad v_{z}=j \omega u_{z} .
$$

(3) Dynamic boundary condition (normal stresses must be continuous) on the interface $\Gamma_{1}$ :

$$
\sigma_{r r}=\Sigma_{r r}, \quad \sigma_{r \theta}=\Sigma_{r \theta}, \quad \sigma_{r z}=\Sigma_{r z} .
$$

(4) On the interface $\Gamma_{2}$ at the outer cylinder the normal stress is equal to zero:

$$
\sigma_{r r}=\sigma_{r \theta}=\sigma_{r z}=0 .
$$

Combining these boundary conditions with (34)-(35) and taking into account (7)-(9) and (32) yields for each mode number $(n, m)$ the following linear system:

$$
[\mathbf{M}]\{\mathbf{y}\}=\{0\},
$$

where $\{\mathbf{y}\}=\{A B C D E F a b c d e f\}^{T}$ and $[\mathbf{M}]$ is an twelfth-order operator matrix, which is given in Appendix C. For nontrivial solution, the determinant of the matrix $\mathbf{M}$ must be equal to zero:

$$
\operatorname{det}[\mathbf{M}]=0 .
$$

This equation indicates a relationship between the dynamic fluid viscosity $\eta$, density of the fluid $\rho$, and the elastic constants. The roots of (41) give the infinite natural frequencies $\omega$.

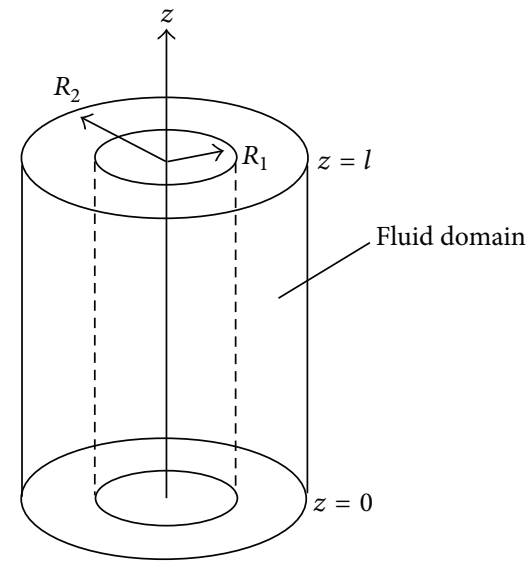

FIGURE 1: Configuration of the viscous oscillations of a cylindrical incompressible fluid of length $l$ in circular cylindrical coordinate system $(r, \theta, z) . R_{1}$ and $R_{2}$ are the inner and outer radius of fluid domain, respectively.



Figure 2: Geometry of fluid domain.

\section{Analytical, Numerical Results and Validation}

Numerical calculations were performed on the example of the hollow cylinder (Figures 1, 2, and 3) with $l=0.15$ [m], and different dimensions such as the inner radius $R_{1}=0.07[\mathrm{~m}]$ and the outer radius $R_{2}=0.09[\mathrm{~m}]$ of fluid domain are used. The fluid used in the hollow cylinder for which density of $920\left[\mathrm{~kg} \cdot \mathrm{m}^{-3}\right]$ and dynamic's viscosity of $0.1[\mathrm{~Pa} \cdot \mathrm{s}]$ is assumed. The following values of parameters of the the elastic solid are in contact with a viscous liquid were assumed: $\rho_{s}=$ $1150\left[\mathrm{~kg} / \mathrm{m}^{3}\right], v_{s}=0.48, E=3 \cdot 10^{5}[\mathrm{~Pa}]$, and the inner radius of elastic solid $R_{0}=0.068[\mathrm{~m}]$.

With the derived eigenfrequency equations, natural frequencies $\omega_{n m}=\operatorname{Im}\left(\nu \alpha_{n m}^{2} j\right)$ for each pair of $(n, m)$ are calculated in the software Mathematica. $m$ and $n$ denote the mode in axial and azimuthal (propagating clockwise around the vortex) direction, respectively. To validate the analytical results, the natural frequencies and mode shapes are also computed using Comsol Multiphysics FEM Simulation Software. The natural frequencies are computed directly from 


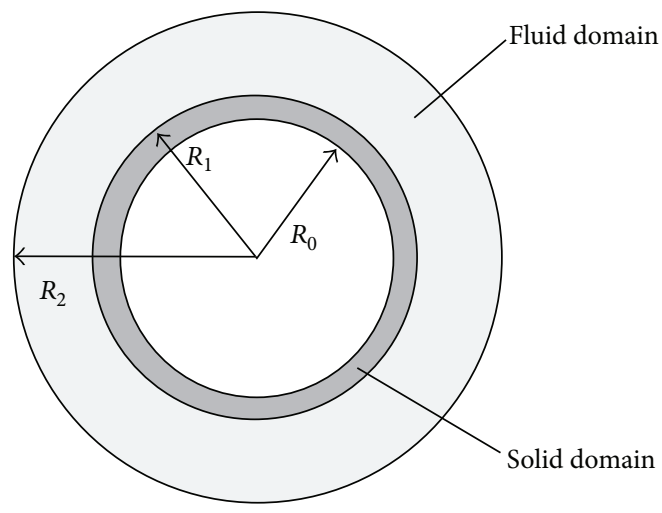

FIgURE 3: Geometry of fluid-solid interation model. $R_{0}$ and $R_{1}$ are the inner and outer radius of solid domain, respectively.

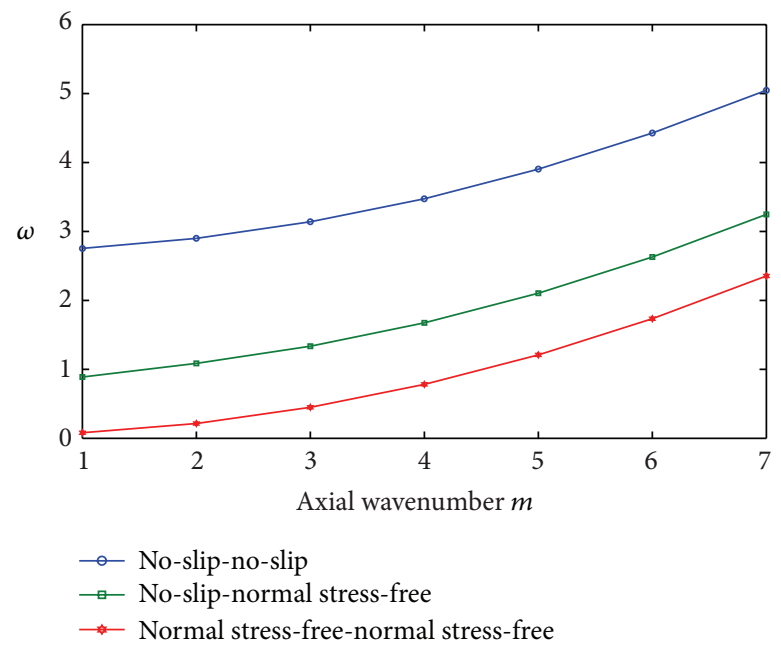

Figure 4: Variations of flexural eigenmode for different boundary conditions.

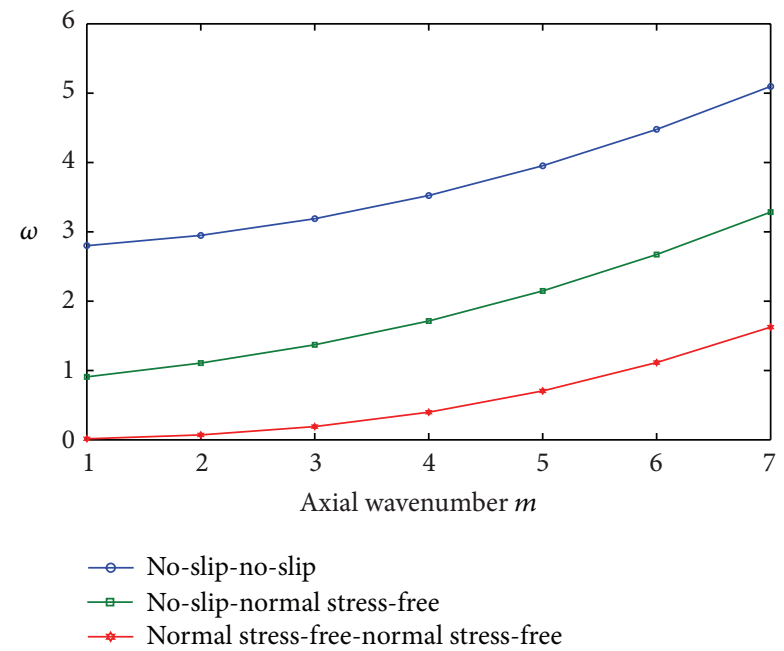

FIGURE 5: Variations of breathing eigenmode for different boundary conditions.



FIGURE 6: Variations of torsional eigenmode for different boundary conditions.

TABLE 1: Natural frequencies $\omega[\mathrm{rd} / \mathrm{s}]$ for various mode shapes in the case of "no-slip-no-slip" boundary conditions.

\begin{tabular}{lllll}
\hline No. & $(n, m)$ & Present & FEM & Mode shape \\
\hline 1 & $(0,0)$ & 2.694 & 2.694 & Torsional \\
2 & $(0,1)$ & 2.742 & 2.742 & Torsional \\
3 & $(1,1)$ & 2.755 & 2.755 & Flexural \\
4 & $(2,1)$ & 2.800 & 2.800 & Breathing \\
5 & $(3,1)$ & 2.882 & 2.882 & Breathing \\
6 & $(0,2)$ & 2.885 & 2.885 & Torsional \\
7 & $(1,2)$ & 2.900 & 2.900 & Flexural \\
8 & $(2,2)$ & 2.948 & 2.948 & Breathing \\
9 & $(4,1)$ & 3.000 & 3.000 & Breathing \\
10 & $(3,2)$ & 3.030 & 3.030 & Breathing \\
11 & $(0,3)$ & 3.124 & 3.124 & Torsional \\
12 & $(1,3)$ & 3.140 & 3.140 & Flexural \\
13 & $(4,2)$ & 3.147 & 3.147 & Breathing \\
14 & $(5,1)$ & 3.153 & 3.153 & Breathing \\
15 & $(2,3)$ & 3.189 & 3.189 & Breathing \\
16 & $(3,3)$ & 3.271 & 3.271 & Breathing \\
17 & $(5,2)$ & 3.299 & 3.299 & Breathing \\
18 & $(6,1)$ & 3.341 & 3.341 & Breathing \\
\hline
\end{tabular}

(24) for torsion vibration. For longitudinal vibration, (28) can be used to determine the corresponding natural frequencies.

Tables 1 and 2 show the comparison of the first 18 natural frequencies and the corresponding mode shapes of viscous fluid by FEM and the present method (see (16) and (20)). For example in the case of "no-slip-normal stress-free" boundary conditions, in the first 18 natural frequencies, four correspond to flexural vibration $(n=1)$, nine to breathing vibration $(n \geqslant 2)$, four to torsional vibration, and one to longitudinal vibration. The very good agreement is observed between the results of the present method and those of FEM and the relative difference ((FEM-Present)/Present) is $\leqslant 1 \%$. This shows that the algorithm implemented in Comsol 


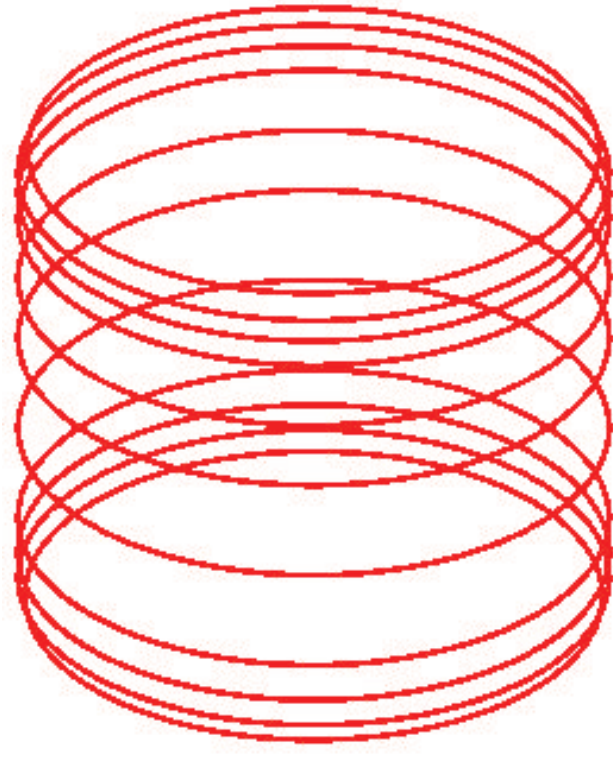

(a) $(n, m)=(0,1)$

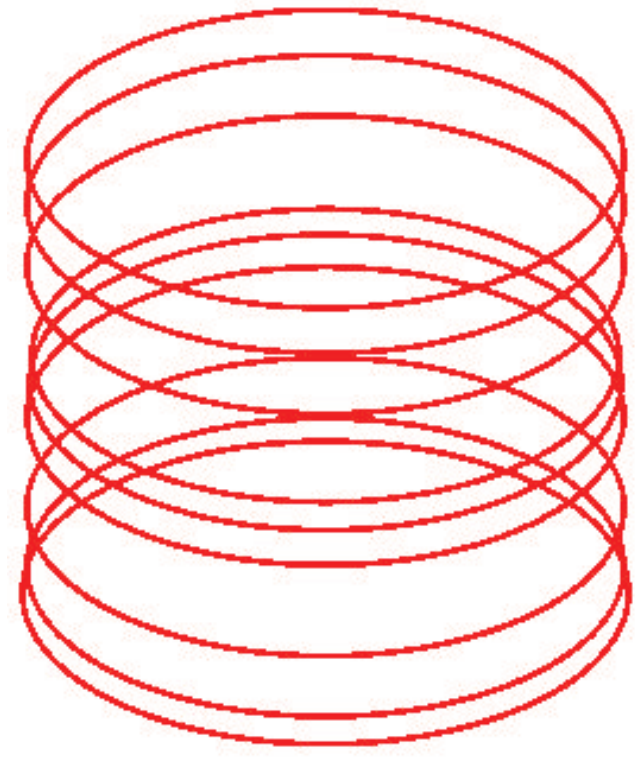

(b) $(n, m)=(0,2)$

Figure 7: The torsional modal shapes of $(n, m)$ : streamlines of components of velocity field.

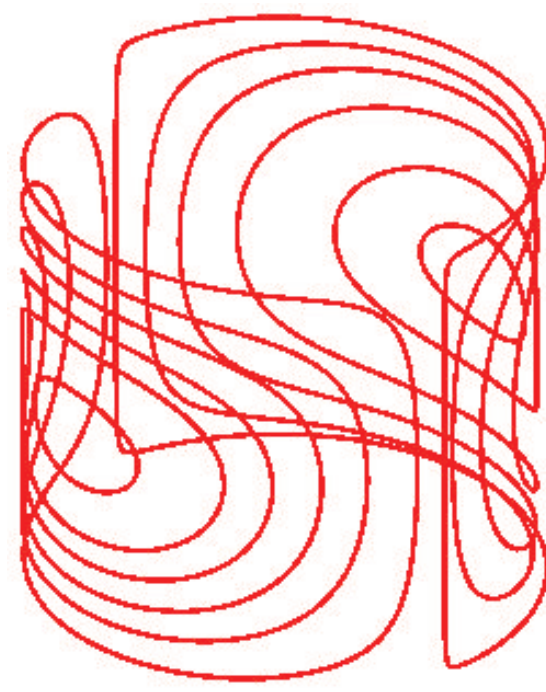

(a) $(n, m)=(1,1)$



(b) $(n, m)=(1,2)$

FIgURE 8: The flexural modal shapes of $(1, m)$ : streamlines of components of velocity field.

Multiphysics [26, 27] software for numerical computation is highly reliable and accurate. This algorithm is based on the UMFPACK method [28]. Is the attention to use the numerical formulation in future for more general geometries.

Tables 1 and 2 and Figures 4, 5, and 6 show that natural frequencies are very sensitive to the nature of the boundary conditions. It is seen that the effect of the free surface is very interesting and decrease the frequency of a fluid confined in a rigid cylinder.

Figures 7, 8, and 9 show, respectively, the two modal shapes of the torsional, flexural, and breathing vibrations. The modal shape can be regarded as the mode $(n, m)$, where $n$ is the modal number in the circumferential direction and $m$ is the modal number in the axial direction. The modal shapes are not in order with the parameters $n$ and $m$. This feature of cylindrical vibration is different from that of beam vibration in which the order increases with the modal parameter. Therefore in the vibration of the cylinder, one should be careful to find the right mode of the vibration.

In this paper, the effects of boundary conditions and of cylindrical parameters on the natural frequencies of cylindrical viscous fluid are presented with the present method. In these studies, investigations are carried out to study the effects of circumferential mode $n$, axial mode $m$, and fluid thickness 


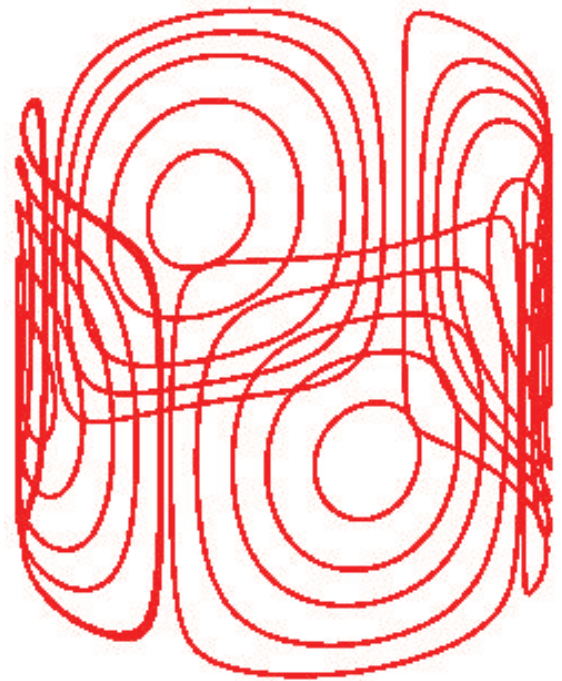

(a) $(n, m)=(2,1)$

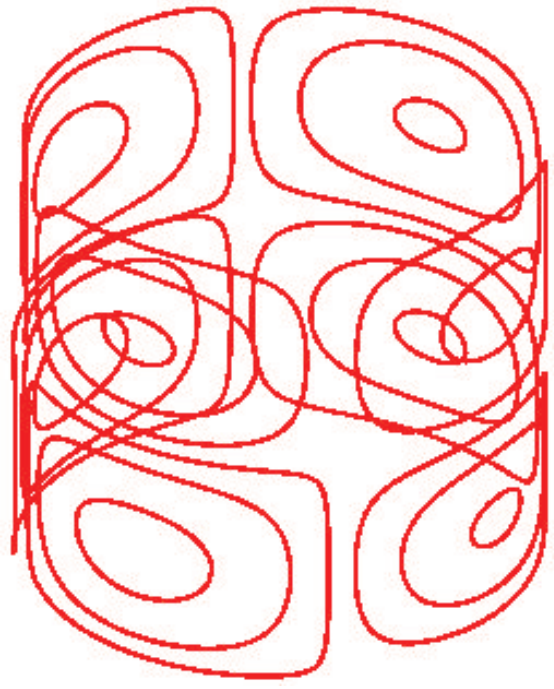

(b) $(n, m)=(2,2)$

FIgURE 9: The breathing modal shapes of $(2, m)$ : streamlines of components of velocity field.

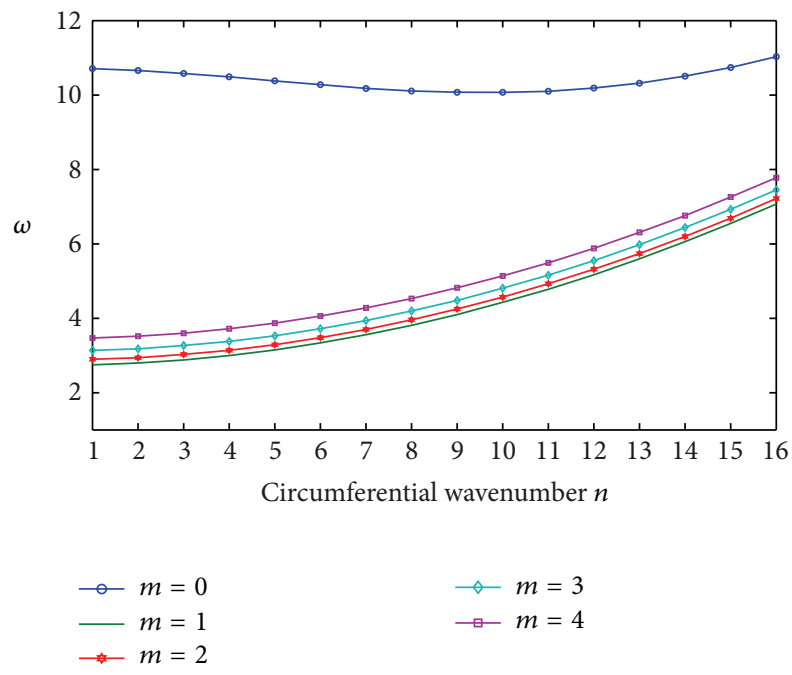

FIGURE 10: Variations of the natural frequencies $\omega$ for different values $m$ with the mode $m$ with the mode $n$.

$e=R_{2}-R_{1}$ on the frequencies. Influence of viscosity on the natural frequencies of an elastic solid is also investigated.

First, one investigates how the natural frequencies vary with the axial mode $m$. Figure 10 shows that the natural frequencies increase as the axial mode $m$ increases except when $m=0$. This value of $m$ corresponds to 2D problem of viscous oscillations.

Secondly, one investigates how the frequencies vary with the fluid thickness. Figures 11, 12, and 13 show that the fluid thickness has a strong influence on the natural frequencies.

Thirdly, one investigates how the dense fluid (added mass) affects the natural frequencies. Tables 3 and 4 show the coupled and uncoupled natural frequencies varying with circumferential and axial mode $(n, m)$. As $n$ increases, the

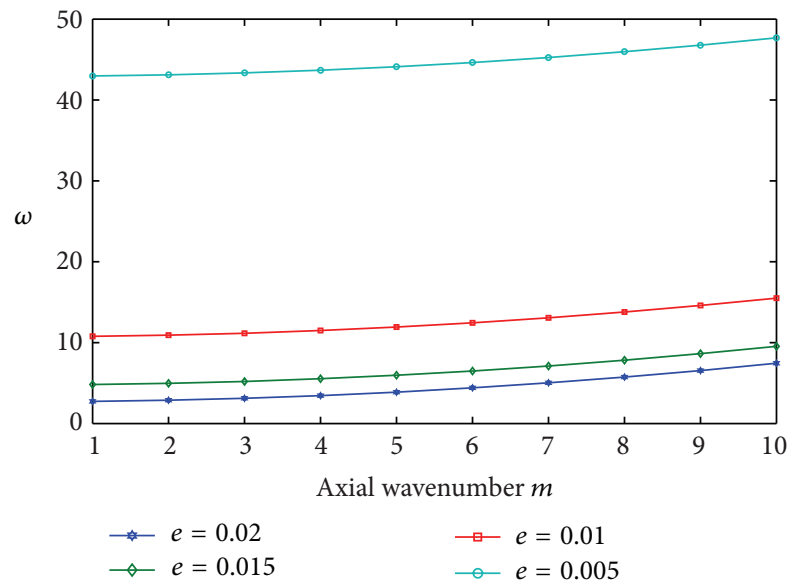

FIGURE 11: Variations of the torsional eigenmode $(n=0) \omega$ for different fluid's thickness $e$ with the mode $m$.

difference between the coupled and uncoupled natural frequencies increases. Figures 14 and 15 show that the presence of fluid has no influence on the modal shapes of an elastic solid.

\section{Conclusion}

We have presented an analytic solution for the Stokes eigenmodes of a viscous incompressible cylindrical fluid. Using the Helmholtz decomposition for the velocity field, we obtain an eigenvalue problem for $\omega$. The analytical results are in very good agreement with FEM results. This analytic method clearly distinguishes between the potential and rotational components and the contributions of each to various flow variables can be analyzed if one wishes so. The present solution is for cylindrical viscous fluid and is not limited to 


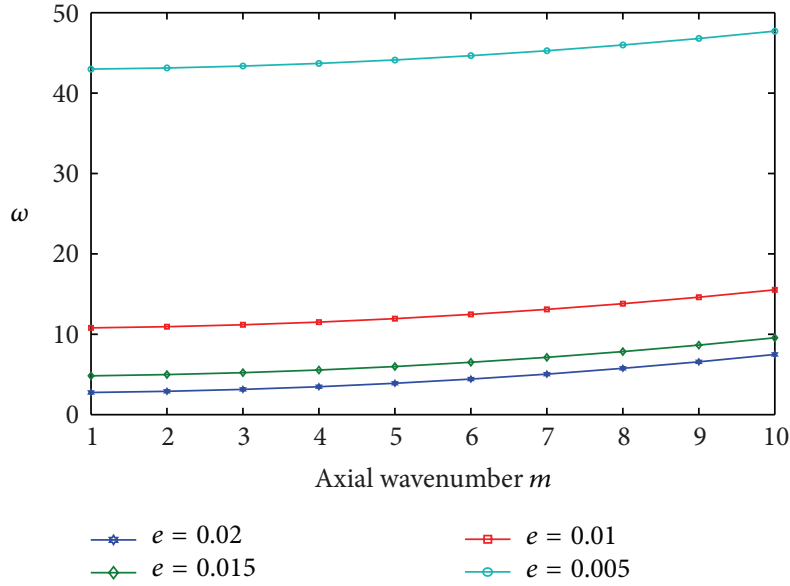

FIGURE 12: Variations of the flexural eigenmode $(n=1) \omega$ for different fluid's thickness $e$ with the mode $m$.

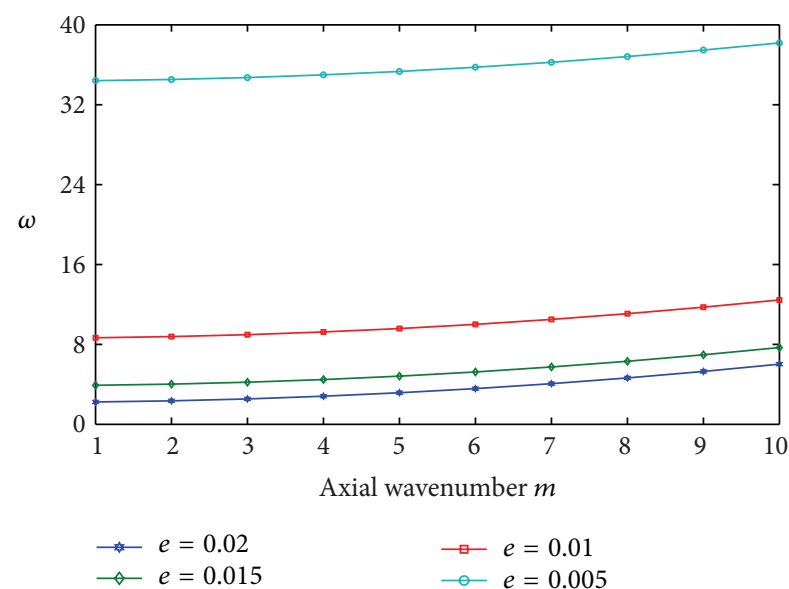

FIGURE 13: Variations of the breathing eigenmode $(n=2) \omega$ for different fluid's thickness $e$ with the mode $m$.

a cylindrical shape. The same scalar potentials can be used to obtain stokes eigenmodes in the case of a spherical geometry. Finally, a knowledge of the stokes eigenmodes of viscous fluid is likely to be of use in performing a dynamic analysis by modal projection method.

\section{Appendices}

A.

The matrix $\mathbf{M}_{1}$ in (16) is defined as follows:

$$
\mathbf{M}_{1}=\left[\begin{array}{cccccc}
a_{11} & a_{12} & a_{13} & a_{14} & a_{15} & a_{16} \\
a_{21} & a_{22} & a_{23} & a_{24} & a_{25} & a_{26} \\
a_{31} & a_{32} & 0 & 0 & a_{35} & a_{36} \\
a_{41} & a_{42} & a_{43} & a_{44} & a_{45} & a_{46} \\
a_{51} & a_{52} & a_{53} & a_{54} & a_{55} & a_{56} \\
a_{61} & a_{62} & 0 & 0 & a_{65} & a_{66}
\end{array}\right],
$$

TABLE 2: Natural nfrequencies $\omega[\mathrm{rd} / \mathrm{s}$ ] for various mode shapes in the case of "no-slip-normal stress-free" boundary conditions.

\begin{tabular}{lcccc}
\hline No. & $(n, m)$ & Present & FEM & Mode shape \\
\hline 1 & $(1,1)$ & 0.889 & 0.889 & Flexural \\
2 & $(0,0)$ & 0.898 & 0.898 & Torsional \\
3 & $(2,1)$ & 0.908 & 0.908 & Breathing \\
4 & $(0,1)$ & 0.946 & 0.946 & Torsional \\
5 & $(0,1)$ & 0.951 & 0.951 & Longitudinal \\
6 & $(3,1)$ & 0.984 & 0.984 & Breathing \\
7 & $(1,0)$ & 0.990 & 0.990 & Flexural \\
8 & $(1,2)$ & 1.087 & 1.087 & Flexural \\
9 & $(0,2)$ & 1.089 & 1.089 & Torsional \\
10 & $(2,2)$ & 1.1070 & 1.1070 & Breathing \\
11 & $(4,1)$ & 1.1073 & 1.1073 & Breathing \\
12 & $(1,1)$ & 1.113 & 1.113 & Flexural \\
13 & $(3,2)$ & 1.171 & 1.171 & Breathing \\
14 & $(2,0)$ & 1.251 & 1.251 & Breathing \\
15 & $(5,1)$ & 1.271 & 1.271 & Breathing \\
16 & $(4,2)$ & 1.281 & 1.281 & Breathing \\
17 & $(0,3)$ & 1.327 & 1.327 & Torsional \\
18 & $(1,3)$ & 1.337 & 1.337 & Flexural \\
\hline
\end{tabular}

TABLE 3: Uncoupled natural frequencies $\omega[\mathrm{rd} / \mathrm{s}]$ for various mode shapes of elastic solid.

\begin{tabular}{llccc}
\hline No. & $(n, m)$ & Present & FEM & Mode shape \\
\hline 1 & $(2,0)$ & 0.952 & 0.952 & Breathing \\
2 & $(3,0)$ & 2.693 & 2.693 & Breathing \\
3 & $(4,0)$ & 5.157 & 5.157 & Breathing \\
4 & $(4,1)$ & 7.145 & 7.145 & Breathing \\
5 & $(3,1)$ & 7.389 & 7.389 & Breathing \\
6 & $(5,0)$ & 8.326 & 8.326 & Breathing \\
7 & $(5,1)$ & 9.431 & 9.431 & Breathing \\
8 & $(2,1)$ & 11.456 & 11.456 & Breathing \\
9 & $(6,0)$ & 12.190 & 12.190 & Breathing \\
10 & $(6,1)$ & 13.035 & 13.035 & Breathing \\
11 & $(5,2)$ & 14.379 & 14.379 & Breathing \\
12 & $(4,2)$ & 14.700 & 14.700 & Breathing \\
13 & $(6,2)$ & 16.504 & 16.504 & Breathing \\
14 & $(7,0)$ & 16.739 & 16.739 & Breathing \\
15 & $(7,1)$ & 17.500 & 17.500 & Breathing \\
16 & $(3,2)$ & 18.005 & 18.005 & Breathing \\
17 & $(7,2)$ & 20.270 & 20.270 & Breathing \\
18 & $(1,1)$ & 20.289 & 20.289 & Flexural \\
\hline
\end{tabular}

where

$$
\begin{gathered}
a_{11}=I_{n}^{\prime}\left(k_{z} R_{1}\right), \quad a_{12}=K_{n}^{\prime}\left(k_{z} R_{1}\right), \\
a_{13}=-\frac{n}{R_{1}} J_{n}\left(k_{\psi} R_{1}\right),
\end{gathered}
$$






(a) $\omega=0.952$

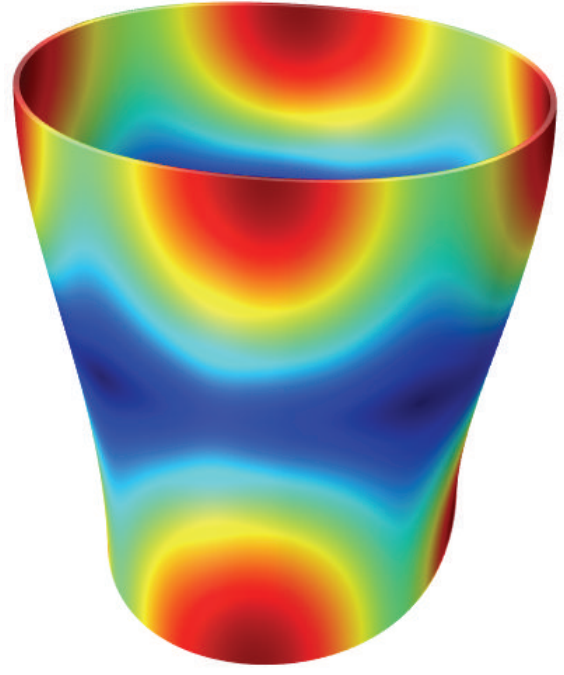

(c) $\omega=11.456$

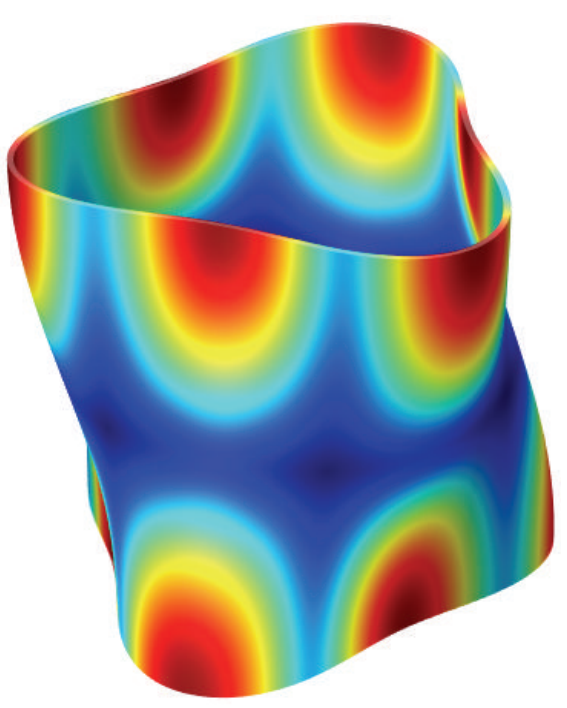

(b) $\omega=7.389$

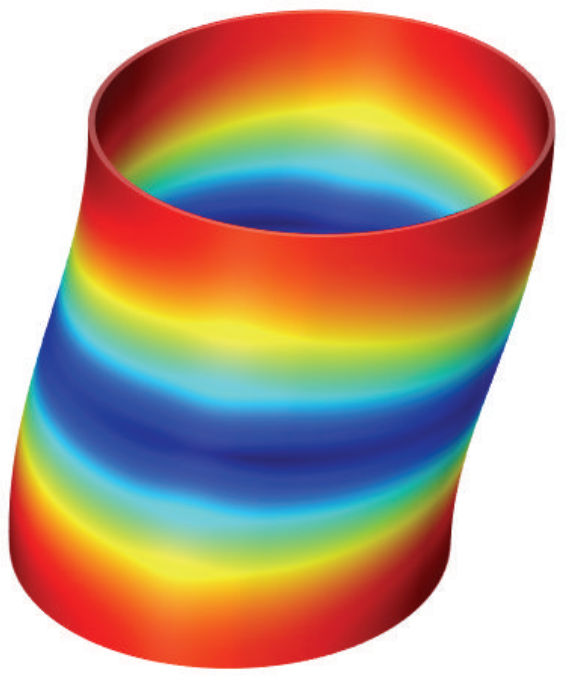

(d) $\omega=20.289$

FIGURE 14: The modal shapes $(n, m)$ of elastic solid without fluid: the colours pertain to the displacement filed.

$$
\begin{aligned}
a_{14}=-\frac{n}{R_{1}} Y_{n}\left(k_{\psi} R_{1}\right), & \\
a_{15}=k_{z} J_{n}^{\prime}\left(k_{\psi} R_{1}\right), & a_{16}=k_{z} Y_{n}^{\prime}\left(k_{\psi} R_{1}\right), \\
a_{21}=\frac{n}{R_{1}} I_{n}\left(k_{z} R_{1}\right), & a_{22}=\frac{n}{R_{1}} K_{n}\left(k_{z} R_{1}\right), \\
a_{23}=-J_{n}^{\prime}\left(k_{\psi} R_{1}\right), & a_{24}=-Y_{n}^{\prime}\left(k_{\psi} R_{1}\right), \\
a_{25}=\frac{n k_{z}}{R_{1}} J_{n}\left(k_{\psi} R_{1}\right), & a_{26}=\frac{n k_{z}}{R_{1}} Y_{n}\left(k_{\psi} R_{1}\right), \\
a_{31}=-k_{z} I_{n}\left(k_{z} R_{1}\right), & a_{32}=-k_{z} K_{n}\left(k_{z} R_{1}\right), \\
a_{35}=k_{\psi}^{2} J_{n}\left(k_{\psi} R_{1}\right), & a_{36}=k_{\psi}^{2} Y_{n}\left(k_{\psi} R_{1}\right),
\end{aligned}
$$

$$
\begin{gathered}
a_{41}=I_{n}^{\prime}\left(k_{z} R_{2}\right), \quad a_{42}=K_{n}^{\prime}\left(k_{z} R_{2}\right), \\
a_{43}=-\frac{n}{R_{2}} J_{n}\left(k_{\psi} R_{2}\right), \\
a_{44}=-\frac{n}{R_{2}} Y_{n}\left(k_{\psi} R_{2}\right), \\
a_{45}=k_{z} J_{n}^{\prime}\left(k_{\psi} R_{2}\right), \quad a_{46}=k_{z} Y_{n}^{\prime}\left(k_{\psi} R_{2}\right), \\
a_{51}=\frac{n}{R_{2}} I_{n}\left(k_{z} R_{2}\right), \quad a_{52}=\frac{n}{R_{2}} K_{n}\left(k_{z} R_{2}\right), \\
a_{53}=-J_{n}^{\prime}\left(k_{\psi} R_{2}\right), \quad a_{54}=-Y_{n}^{\prime}\left(k_{\psi} R_{2}\right), \\
a_{55}=\frac{n k_{z}}{R_{2}} J_{n}\left(k_{\psi} R_{2}\right),
\end{gathered}
$$


TABLE 4: Coupled natural nfrequencies $\omega[\mathrm{rd} / \mathrm{s}]$ for various mode shapes.

\begin{tabular}{lcccc}
\hline No. & $(n, m)$ & Present & FEM & Mode shape \\
\hline 1 & $(0,1)$ & 0.535 & 0.535 & Torsional \\
2 & $(1,1)$ & 0.566 & 0.566 & Flexural \\
3 & $(2,1)$ & 0.645 & 0.645 & Breathing \\
4 & $(0,2)$ & 0.678 & 7.145 & Torsional \\
5 & $(1,2)$ & 0.699 & 0.699 & Flexural \\
6 & $(2,0)$ & 0.702 & 0.702 & Breathing \\
7 & $(3,1)$ & 0.749 & 0.749 & Breathing \\
8 & $(2,2)$ & 0.760 & 0.760 & Breathing \\
9 & $(0,1)$ & 0.780 & 0.780 & Longitudinal \\
10 & $(1,1)$ & 0.813 & 0.813 & Flexural \\
11 & $(3,2)$ & 0.854 & 0.854 & Breathing \\
12 & $(4,1)$ & 0.872 & 0.872 & Breathing \\
13 & $(0,3)$ & 0.917 & 0.917 & Torsional \\
14 & $(3,0)$ & 0.924 & 0.924 & Breathing \\
15 & $(1,3)$ & 0.935 & 0.935 & Flexural \\
16 & $(4,2)$ & 0.978 & 0.978 & Breathing \\
17 & $(2,3)$ & 0.988 & 0.988 & Breathing \\
18 & $(5,1)$ & 1.019 & 1.019 & Breathing \\
\hline
\end{tabular}

$$
\begin{array}{ll}
a_{61}=-k_{z} I_{n}\left(k_{z} R_{2}\right), & a_{62}=-k_{z} K_{n}\left(k_{z} R_{2}\right), \\
a_{65}=k_{\psi}^{2} J_{n}\left(k_{\psi} R_{2}\right), & a_{66}=k_{\psi}^{2} Y_{n}\left(k_{\psi} R_{2}\right) .
\end{array}
$$

\section{B.}

The matrix $\mathbf{M}_{2}$ in (20) is defined as follows:

$$
\mathbf{M}_{2}=\left[\begin{array}{llllll}
b_{11} & b_{12} & b_{13} & b_{14} & b_{15} & b_{16} \\
b_{21} & b_{22} & b_{23} & b_{24} & b_{25} & b_{26} \\
b_{31} & b_{32} & b_{33} & b_{34} & b_{35} & b_{36} \\
a_{41} & a_{42} & a_{43} & a_{44} & a_{45} & a_{46} \\
a_{51} & a_{52} & a_{53} & a_{54} & a_{55} & a_{56} \\
a_{61} & a_{62} & 0 & 0 & a_{65} & a_{66}
\end{array}\right],
$$

where

$$
\begin{gathered}
b_{11}=\eta\left[2 I_{n}^{\prime \prime}\left(k_{z} R_{1}\right)-\alpha^{2} I_{n}\left(k_{z} R_{1}\right)\right], \\
b_{12}=\eta\left[2 K_{n}^{\prime \prime}\left(k_{z} R_{1}\right)-\alpha^{2} K_{n}\left(k_{z} R_{1}\right)\right], \\
b_{13}=\frac{2 \eta n}{R_{1}^{2}}\left[J_{n}\left(k_{\psi} R_{1}\right)-R_{1} J_{n}^{\prime}\left(k_{\psi} R_{1}\right)\right], \\
b_{14}=\frac{2 \eta n}{R_{1}^{2}}\left[Y_{n}\left(k_{\psi} R_{1}\right)-R_{1} Y_{n}^{\prime}\left(k_{\psi} R_{1}\right)\right], \\
b_{15}=2 \eta k_{z} J_{n}^{\prime \prime}\left(k_{\psi} R_{1}\right), \\
b_{16}=2 \eta k_{z} Y_{n}^{\prime \prime}\left(k_{\psi} R_{1}\right),
\end{gathered}
$$

$$
\begin{gathered}
b_{21}=\frac{2 \eta n}{R_{1}^{2}}\left[R_{1} I_{n}^{\prime}\left(k_{z} R_{1}\right)-I_{n}\left(k_{z} R_{1}\right)\right], \\
b_{22}=\frac{2 \eta n}{R_{1}^{2}}\left[R_{1} K_{n}^{\prime}\left(k_{z} R_{1}\right)-K_{n}\left(k_{z} R_{1}\right)\right], \\
b_{23}=-\eta\left[2 J_{n}^{\prime \prime}\left(k_{\psi} R_{1}\right)+k_{\psi}^{2} J_{n}\left(k_{\psi} R_{1}\right)\right], \\
b_{24}=-\eta\left[2 Y_{n}^{\prime \prime}\left(k_{\psi} R_{1}\right)+k_{\psi}^{2} Y_{n}\left(k_{\psi} R_{1}\right)\right], \\
b_{25}=\frac{2 \eta n k_{z}}{R_{1}^{2}}\left[R_{1} I_{n}^{\prime}\left(k_{z} R_{1}\right)-I_{n}\left(k_{z} R_{1}\right)\right], \\
b_{26}=\frac{2 \eta n k_{z}}{R_{1}^{2}}\left[R_{1} K_{n}^{\prime}\left(k_{z} R_{1}\right)-K_{n}\left(k_{z} R_{1}\right)\right], \\
b_{31}=-2 \eta k_{z} I_{n}^{\prime}\left(k_{z} R_{1}\right), \\
b_{32}=-2 \eta k_{z} K_{n}^{\prime}\left(k_{z} R_{1}\right), \\
b_{33}=\frac{\eta n k_{z}}{R_{1}} J_{n}\left(k_{\psi} R_{1}\right), \\
b_{34}=\frac{\eta n k_{z}}{R_{1}} Y_{n}\left(k_{\psi} R_{1}\right), \\
b_{35}=\eta\left(k_{\psi}^{2}-k_{z}^{2}\right) J_{n}^{\prime}\left(k_{\psi} R_{1}\right), \\
b_{36}=\eta\left(k_{\psi}^{2}-k_{z}^{2}\right) Y_{n}^{\prime}\left(k_{\psi} R_{1}\right) .
\end{gathered}
$$

C.

The operator matrix $\mathbf{M}$ in (41) is defined as follows:

$$
\begin{aligned}
& M=\left[\begin{array}{ll}
A & B \\
C & D
\end{array}\right], \\
& \mathbf{A}=\left[\begin{array}{cccccc}
0 & 0 & 0 & 0 & 0 & 0 \\
0 & 0 & 0 & 0 & 0 & 0 \\
0 & 0 & 0 & 0 & 0 & 0 \\
b_{11} & b_{12} & b_{13} & b_{14} & b_{15} & b_{16} \\
b_{21} & b_{22} & b_{23} & b_{24} & b_{25} & b_{26} \\
b_{31} & b_{32} & b_{33} & b_{34} & b_{35} & b_{36}
\end{array}\right], \\
& \mathbf{B}=\left[\begin{array}{llllll}
c_{17} & c_{18} & c_{19} & c_{110} & c_{111} & c_{112} \\
c_{27} & c_{28} & c_{29} & c_{210} & c_{211} & c_{212} \\
c_{37} & c_{38} & c_{39} & c_{310} & c_{311} & c_{312} \\
c_{47} & c_{48} & c_{49} & c_{410} & c_{411} & c_{412} \\
c_{57} & c_{58} & c_{59} & c_{510} & c_{511} & c_{512} \\
c_{67} & c_{68} & c_{69} & c_{6} & c_{611} & c_{612}
\end{array}\right],
\end{aligned}
$$




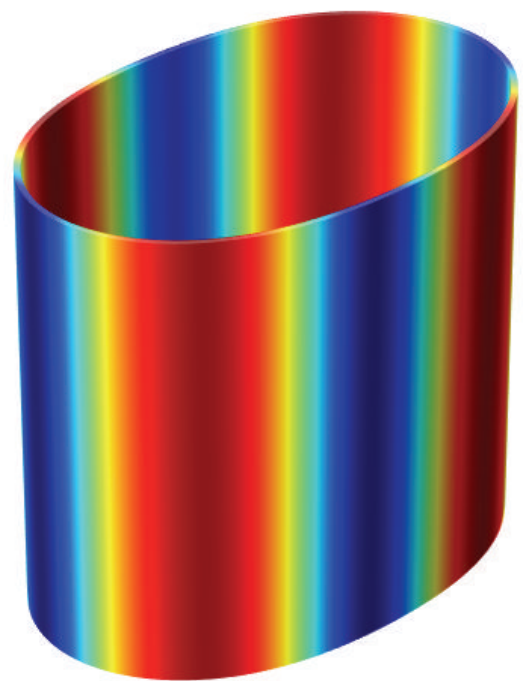

(a) $\omega=0.702$

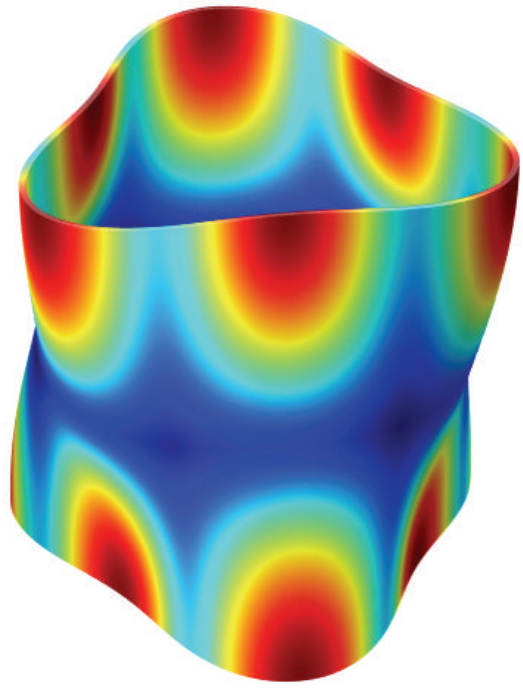

(b) $\omega=0.749$

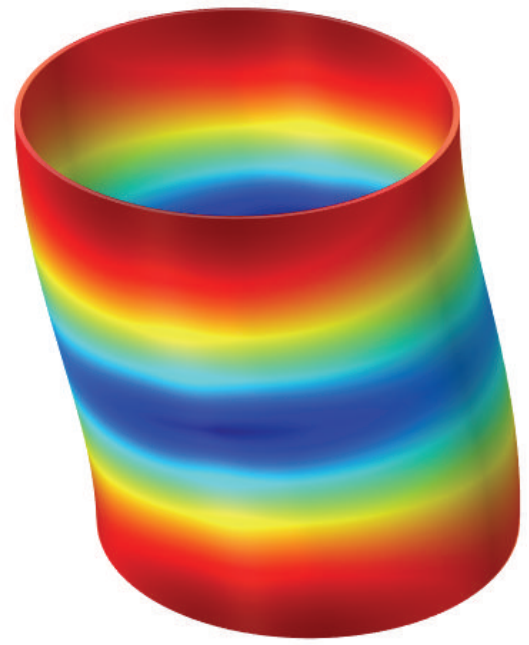

(d) $\omega=0.813$

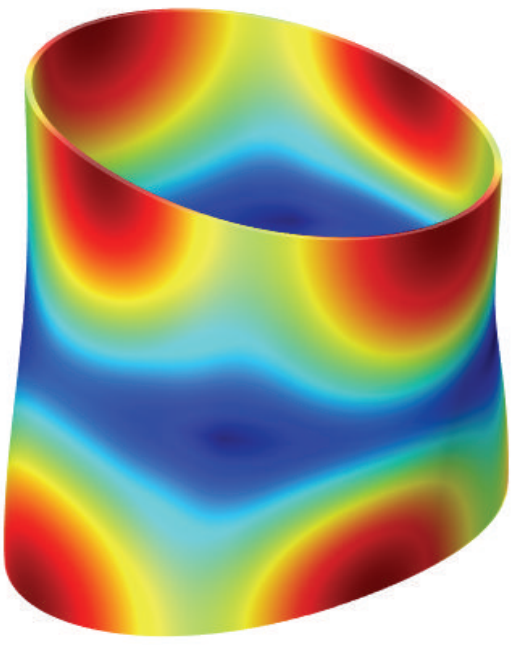

(c) $\omega=0.645$

FIGURE 15: The modal shapes $(n, m)$ of elastic solid with fluid: the colours pertain to the displacement filed.

$$
\begin{aligned}
\mathbf{C} & =\left[\begin{array}{cccccc}
a_{11} & a_{12} & a_{13} & a_{14} & a_{15} & a_{16} \\
a_{21} & a_{22} & a_{23} & a_{24} & a_{25} & a_{26} \\
a_{31} & a_{32} & 0 & 0 & a_{35} & a_{36} \\
c_{101} & c_{102} & c_{103} & c_{104} & c_{105} & c_{106} \\
c_{11} & c_{112} & c_{113} & c_{114} & c_{115} & c_{116} \\
c_{121} & c_{122} & c_{123} & c_{124} & c_{125} & c_{126}
\end{array}\right], \\
\mathbf{D} & =\left[\begin{array}{ccccccc}
c_{77} & c_{78} & c_{79} & c_{710} & c_{711} & c_{712} \\
c_{87} & c_{88} & c_{89} & c_{810} & c_{811} & c_{812} \\
c_{97} & c_{98} & 0 & 0 & c_{911} & c_{912} \\
0 & 0 & 0 & 0 & 0 & 0 \\
0 & 0 & 0 & 0 & 0 & 0 \\
0 & 0 & 0 & 0 & 0 & 0
\end{array}\right],
\end{aligned}
$$

where

$$
\begin{gathered}
c_{17}=2 \mu\left[I_{n}^{\prime \prime}\left(k_{\Phi} R_{0}\right)+\frac{\lambda v_{f}^{2} \alpha^{4}}{2 \mu c_{L}^{2}} I_{n}\left(k_{\Phi} R_{0}\right)\right], \\
c_{18}=2 \mu\left[K_{n}^{\prime \prime}\left(k_{\Phi} R_{0}\right)+\frac{\lambda v_{f}^{2} \alpha^{4}}{2 \mu c_{L}^{2}} K_{n}\left(k_{\Phi} R_{0}\right)\right], \\
c_{19}=\frac{2 \mu n}{R_{0}^{2}}\left[I_{n}\left(k_{\Psi} R_{0}\right)-R_{0} I_{n}^{\prime}\left(k_{\Psi} R_{0}\right)\right], \\
c_{110}=\frac{2 \mu n}{R_{0}^{2}}\left[K_{n}\left(k_{\Psi} R_{0}\right)-R_{0} K_{n}^{\prime}\left(k_{\Psi} R_{0}\right)\right],
\end{gathered}
$$




$$
\begin{aligned}
& c_{111}=2 \mu k_{z} I_{n}^{\prime \prime}\left(k_{\Psi} R_{0}\right), \\
& c_{112}=2 \mu k_{z} K_{n}^{\prime \prime}\left(k_{\Psi} R_{0}\right) \text {, } \\
& c_{27}=\frac{2 \mu n}{R_{0}^{2}}\left[R_{0} I_{n}^{\prime}\left(k_{\Phi} R_{0}\right)-I_{n}\left(k_{\Phi} R_{0}\right)\right], \\
& c_{28}=\frac{2 \mu n}{R_{0}^{2}}\left[R_{0} K_{n}^{\prime}\left(k_{\Phi} R_{0}\right)-K_{n}\left(k_{\Phi} R_{0}\right)\right], \\
& c_{29}=\mu\left[k_{\Psi}^{2} I_{n}\left(k_{\Psi} R_{0}\right)-2 I_{n}^{\prime \prime}\left(k_{\Psi} R_{0}\right)\right], \\
& c_{210}=\mu\left[k_{\Psi}^{2} K_{n}\left(k_{\Psi} R_{0}\right)-2 K_{n}^{\prime \prime}\left(k_{\Psi} R_{0}\right)\right], \\
& c_{211}=\frac{2 \mu n k_{z}}{R_{0}^{2}}\left[R_{0} I_{n}^{\prime}\left(k_{\Psi} R_{0}\right)-I_{n}\left(k_{\Psi} R_{0}\right)\right], \\
& c_{212}=\frac{2 \mu n k_{z}}{R_{0}^{2}}\left[R_{0} K_{n}^{\prime}\left(k_{\Psi} R_{0}\right)-K_{n}\left(k_{\Psi} R_{0}\right)\right] \text {, } \\
& c_{37}=-2 \mu k_{z} I_{n}^{\prime}\left(k_{\Phi} R_{0}\right), \\
& c_{38}=-2 \mu k_{z} K_{n}^{\prime}\left(k_{\Phi} R_{0}\right) \text {, } \\
& c_{39}=\frac{\mu n k_{z}}{R_{0}} I_{n}^{\prime}\left(k_{\Psi} R_{0}\right) \text {, } \\
& c_{310}=\frac{\mu n k_{z}}{R_{0}} K_{n}^{\prime}\left(k_{\Psi} R_{0}\right), \\
& c_{311}=-\mu\left(k_{\Psi}^{2}+k_{z}^{2}\right) I_{n}^{\prime}\left(k_{\Psi} R_{0}\right), \\
& c_{312}=-\mu\left(k_{\Psi}^{2}+k_{z}^{2}\right) K_{n}^{\prime}\left(k_{\Psi} R_{0}\right) \text {, } \\
& c_{47}=2 \mu\left[I_{n}^{\prime \prime}\left(k_{\Phi} R_{1}\right)+\frac{\lambda v_{f}^{2} \alpha^{4}}{2 \mu c_{L}^{2}} I_{n}\left(k_{\Phi} R_{1}\right)\right], \\
& c_{48}=2 \mu\left[K_{n}^{\prime \prime}\left(k_{\Phi} R_{1}\right)+\frac{\lambda v_{f}^{2} \alpha^{4}}{2 \mu c_{L}^{2}} K_{n}\left(k_{\Phi} R_{1}\right)\right] \text {, } \\
& c_{49}=\frac{2 \mu n}{R_{1}^{2}}\left[I_{n}\left(k_{\Psi} R_{1}\right)-R_{1} I_{n}^{\prime}\left(k_{\Psi} R_{1}\right)\right], \\
& c_{410}=\frac{2 \mu n}{R_{1}^{2}}\left[K_{n}\left(k_{\Psi} R_{1}\right)-R_{1} K_{n}^{\prime}\left(k_{\Psi} R_{1}\right)\right], \\
& c_{411}=2 \mu k_{z} I_{n}^{\prime \prime}\left(k_{\Psi} R_{1}\right), \\
& c_{412}=2 \mu k_{z} K_{n}^{\prime \prime}\left(k_{\Psi} R_{1}\right) \text {, } \\
& c_{57}=\frac{2 \mu n}{R_{1}^{2}}\left[R_{1} I_{n}^{\prime}\left(k_{\Phi} R_{1}\right)-I_{n}\left(k_{\Phi} R_{1}\right)\right], \\
& c_{58}=\frac{2 \mu n}{R_{1}^{2}}\left[R_{1} K_{n}^{\prime}\left(k_{\Phi} R_{1}\right)-K_{n}\left(k_{\Phi} R_{1}\right)\right], \\
& c_{59}=\mu\left[k_{\Psi}^{2} I_{n}\left(k_{\Psi} R_{1}\right)-2 I_{n}^{\prime \prime}\left(k_{\Psi} R_{1}\right)\right], \\
& c_{510}=\mu\left[k_{\Psi}^{2} K_{n}\left(k_{\Psi} R_{1}\right)-2 K_{n}^{\prime \prime}\left(k_{\Psi} R_{1}\right)\right], \\
& \begin{array}{c}
c_{511}=\frac{2 \mu n k_{z}}{R_{1}^{2}}\left[R_{1} I_{n}^{\prime}\left(k_{\Psi} R_{1}\right)-I_{n}\left(k_{\Psi} R_{1}\right)\right], \\
c_{512=\frac{2 \mu n k_{z}}{R_{1}^{2}}}\left[R_{1} K_{n}^{\prime}\left(k_{\Psi} R_{1}\right)-K_{n}\left(k_{\Psi} R_{1}\right)\right], \\
c_{67}=-2 \mu k_{z} I_{n}^{\prime}\left(k_{\Phi} R_{1}\right), \\
c_{68}=-2 \mu k_{z} K_{n}^{\prime}\left(k_{\Phi} R_{1}\right), \\
c_{69}=\frac{\mu n k_{z}}{R_{1}} I_{n}^{\prime}\left(k_{\Psi} R_{1}\right), \\
c_{610}=\frac{\mu n k_{z}}{R_{1}} K_{n}^{\prime}\left(k_{\Psi} R_{1}\right),
\end{array} \\
& c_{611}=-\mu\left(k_{\Psi}^{2}+k_{z}^{2}\right) I_{n}^{\prime}\left(k_{\Psi} R_{1}\right), \\
& c_{612}=-\mu\left(k_{\Psi}^{2}+k_{z}^{2}\right) K_{n}^{\prime}\left(k_{\Psi} R_{1}\right) \text {, } \\
& c_{77}=v \alpha^{2} I_{n}^{\prime}\left(k_{\Phi} R_{1}\right) \text {, } \\
& c_{78}=v \alpha^{2} K_{n}^{\prime}\left(k_{\Phi} R_{1}\right) \text {, } \\
& c_{79}=-\frac{v \alpha^{2} n}{R_{1}} I_{n}^{\prime}\left(k_{\Psi} R_{1}\right) \text {, } \\
& c_{710}=-\frac{v \alpha^{2} n}{R_{1}} K_{n}^{\prime}\left(k_{\Psi} R_{1}\right) \text {, } \\
& c_{711}=v \alpha^{2} k_{z} I_{n}^{\prime}\left(k_{\Psi} R_{1}\right) \text {, } \\
& c_{712}=\nu \alpha^{2} k_{z} K_{n}^{\prime}\left(k_{\Psi} R_{1}\right) \text {, } \\
& c_{87}=v \alpha^{2} \frac{n}{R_{1}} I_{n}\left(k_{\Phi} R_{1}\right) \text {, } \\
& c_{88}=v \alpha^{2} \frac{n}{R_{1}} K_{n}\left(k_{\Phi} R_{1}\right) \text {, } \\
& c_{89}=-\nu \alpha^{2} I_{n}^{\prime}\left(k_{\Psi} R_{1}\right) \text {, } \\
& c_{810}=-v \alpha^{2} K_{n}^{\prime}\left(k_{\Psi} R_{1}\right) \text {, } \\
& c_{811}=\nu \alpha^{2} \frac{n k_{z}}{R_{1}} I_{n}\left(k_{\Psi} R_{1}\right) \text {, } \\
& c_{812}=v \alpha^{2} \frac{n k_{z}}{R_{1}} K_{n}\left(k_{\Psi} R_{1}\right) \text {, } \\
& c_{97}=-v \alpha^{2} k_{z} I_{n}\left(k_{\Phi} R_{1}\right) \text {, } \\
& c_{98}=-\nu \alpha^{2} k_{z} K_{n}\left(k_{\Phi} R_{1}\right), \\
& c_{911}=-v \alpha^{2} k_{\Psi}^{2} I_{n}\left(k_{\Psi} R_{1}\right) \text {, } \\
& c_{912}=-v \alpha^{2} k_{\Psi}^{2} K_{n}\left(k_{\Psi} R_{1}\right) \text {, } \\
& c_{101}=\eta\left[2 I_{n}^{\prime \prime}\left(k_{z} R_{2}\right)-\alpha^{2} I_{n}\left(k_{z} R_{2}\right)\right], \\
& c_{102}=\eta\left[2 K_{n}^{\prime \prime}\left(k_{z} R_{2}\right)-\alpha^{2} K_{n}\left(k_{z} R_{2}\right)\right] \text {, }
\end{aligned}
$$




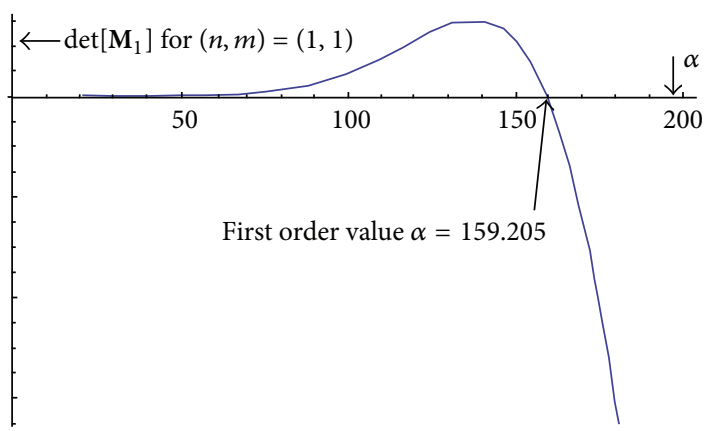

FIGURE 16: Analytical calculation of eigenvalues $\alpha$.

$$
\begin{aligned}
& c_{103}=2 \eta \frac{n}{R_{2}^{2}}\left[J_{n}\left(k_{\psi} R_{2}\right)-R_{2} J_{n}^{\prime}\left(k_{\psi} R_{2}\right)\right] \text {, } \\
& c_{104}=2 \eta \frac{n}{R_{2}^{2}}\left[Y_{n}\left(k_{\psi} R_{2}\right)-R_{2} Y_{n}^{\prime}\left(k_{\psi} R_{2}\right)\right] \text {, } \\
& c_{105}=2 \eta k_{z} J_{n}^{\prime \prime}\left(k_{\psi} R_{2}\right) \text {, } \\
& c_{106}=2 \eta k_{z} Y_{n}^{\prime \prime}\left(k_{\psi} R_{2}\right) \text {, } \\
& c_{111}=2 \eta \frac{n}{R_{2}^{2}}\left[R_{2} I_{n}^{\prime}\left(k_{z} R_{2}\right)-I_{n}\left(k_{z} R_{2}\right)\right], \\
& c_{112}=2 \eta \frac{n}{R_{2}^{2}}\left[R_{2} K_{n}^{\prime}\left(k_{z} R_{2}\right)-K_{n}\left(k_{z} R_{2}\right)\right] \text {, } \\
& c_{113}=-\eta\left[2 J_{n}^{\prime \prime}\left(k_{\psi} R_{2}\right)+k_{\psi}^{2} J_{n}\left(k_{\psi} R_{2}\right)\right] \text {, } \\
& c_{114}=-\eta\left[2 Y_{n}^{\prime \prime}\left(k_{\psi} R_{2}\right)+k_{\psi}^{2} Y_{n}\left(k_{\psi} R_{2}\right)\right] \text {, } \\
& c_{115}=2 \eta \frac{n k_{z}}{R_{2}^{2}}\left[R_{2} I_{n}^{\prime}\left(k_{z} R_{2}\right)-I_{n}\left(k_{z} R_{2}\right)\right] \text {, } \\
& c_{116}=2 \eta \frac{n k_{z}}{R_{2}^{2}}\left[R_{2} K_{n}^{\prime}\left(k_{z} R_{2}\right)-K_{n}\left(k_{z} R_{2}\right)\right] \text {, } \\
& c_{121}=-2 \eta k_{z} I_{n}^{\prime}\left(k_{z} R_{2}\right) \text {, } \\
& c_{122}=-2 \eta k_{z} K_{n}^{\prime}\left(k_{z} R_{2}\right) \text {, } \\
& c_{123}=\eta \frac{n k_{z}}{R_{2}} J_{n}\left(k_{\psi} R_{2}\right) \text {, } \\
& c_{124}=\eta \frac{n k_{z}}{R_{2}} Y_{n}\left(k_{\psi} R_{2}\right) \text {, } \\
& c_{125}=\eta\left(k_{\psi}^{2}-k_{z}^{2}\right) J_{n}^{\prime}\left(k_{\psi} R_{2}\right) \text {, } \\
& c_{126}=\eta\left(k_{\psi}^{2}-k_{z}^{2}\right) Y_{n}^{\prime}\left(k_{\psi} R_{2}\right) \text {. }
\end{aligned}
$$

D.

See Figure 16.

\section{References}

[1] F. Razafimahery, "Ecoulements externes de Stokes dans des domaines non réguliers et condition de Kutta-Joukowski généralisée," Zeitschrift für Angewandte Mathematik und Physik, vol. 54, pp. 125-148, 2003.

[2] H. Bruus, Theoretical Microfluidics, Oxford University Press, New York, NY, USA, 2008.

[3] G. Karniadakis, A. Beskok, and N. Aluru, Microflows and Nanoflows: Fundamentals and Simulation, Springer, 2005.

[4] S. Childress, Mechanics of Swimming and Flying, Cambridge University Press, 1981.

[5] A. S. Ashour, "Propagation of guided waves in a fluid layer bounded by two viscoelastic transversely isotropic solids," Journal of Applied Geophysics, vol. 44, no. 4, pp. 327-336, 2000.

[6] A. S. Ashour, "Wave motion in a viscous fluid-filled fracture," International Journal of Engineering Science, vol. 38, no. 5, pp. 505-515, 2000.

[7] I. Edelman, "On the existence of the low-frequency surface waves in a porous medium," Comptes Rendus, vol. 332, no. 1, pp. 43-49, 2004.

[8] W. Seemann and J. Wauer, "Fluid-structural coupling of vibrating bodies in a surrounding confined liquid," Zeitschrift fur Angewandte Mathematik und Mechanik, vol. 76, no. 2, pp. 67-79, 1996.

[9] S. G. Kadyrov, J. Wauer, and S. V. Sorokin, "A potential technique in the theory of interaction between a structure and a viscous, compressible fluid," Archive of Applied Mechanics, vol. 71, no. 67, pp. 405-417, 2001.

[10] E. Leriche and G. Labrosse, "Vector potential-vorticity relationship for the Stokes flows: application to the Stokes eigenmodes in 2D/3D closed domain," Theoretical and Computational Fluid Dynamics, vol. 21, no. 1, pp. 1-13, 2007.

[11] R. Rodríguez and J. E. Solomin, "The order of convergence of eigenfrequencies in finite element approximations of fluidstructure interaction problems," Mathematics of Computation, vol. 65 , no. 216 , pp. $1463-1475$, 1996.

[12] A. Bermúdez and R. Rodríguez, "Modelling an numerical solution of elastoacoustic vibrations with interface damping," International Journal for Numerical Methods in Engineering, vol. 46, no. 10, pp. 1763-1779, 1999.

[13] G. G. Stokes, "On the effect of the internal friction of fluids on the motion of pendulums," Transactions of the Cambridge Philosophical Society, vol. 9, pp. 8-106, 1851.

[14] H. Lamb, Hydrodynamics, Cambridge University Press, 1932.

[15] D. D. Joseph, "Potential flow of viscous fluids: historical notes," International Journal of Multiphase Flow, vol. 32, no. 3, pp. 285310, 2006.

[16] R. Kidambi, "Oscillatory stokes flow in a cylindrical container," Fluid Dynamics Research, vol. 38, no. 4, pp. 274-294, 2006.

[17] P. N. Shankar, "Three-dimensional eddy structure in a cylindrical container," Journal of Fluid Mechanics, vol. 342, pp. 97-118, 1997.

[18] G. Pontrelli and A. Tatone, "Wave propagation in a fluid flowing through a curved thin-walled elastic tube," Journal of FluidMechanics, vol. 5, pp. 113-133, 1971.

[19] B. Sweetman, M. Xenos, L. Zitella, and A. A. Linninger, "Threedimensional computational prediction of cerebrospinal fluid flow in the human brain," Computers in Biology and Medicine, vol. 41, no. 2, pp. 67-75, 2011. 
[20] S. Gupta, M. Soellinger, P. Boesiger, D. Poulikakos, and V. Kurtcuoglu, "Three-dimensional computational modeling of subject-specific cerebrospinal fluid flow in the subarachnoid space," Journal of Biomechanical Engineering, vol. 131, no. 2, 11 pages, 2009.

[21] V. V. Mokeyev, "On a method for vibration analysis of viscous compressible fluid-structure systems," International Journal for Numerical Methods in Engineering, vol. 59, no. 13, pp. 1703-1723, 2004.

[22] M. Morse and H. Feshbach, Methods of Theoretical Physics, McGraw-Hill, New York, NY, USA, 1946.

[23] R. Ohayon and C. Soize, Structural Acoustics and Vibration, Academic Press, 1997.

[24] M. P. Paidoussis, Fluid-Structure Interactions Slender Structures and Axial Flow, vol. 1, London Academic Press, 1997.

[25] M. P. Paidoussis, Fluid-Structure Interactions Slender Structures and Axial Flow, vol. 2, Elsevier Academic Press, London, UK, 2004.

[26] “Comsol Multiphysics 3.5a," User's Guide, 2008.

[27] "Comsol Multiphysics 3.5a," Reference Guide, 2008.

[28] T. A. Davis, "Algorithm 832: UMFPACK V4.3: an unsymmetricpattern multifrontal method," ACM Transactions on Mathematical Software, vol. 30, no. 2, pp. 196-199, 2004. 

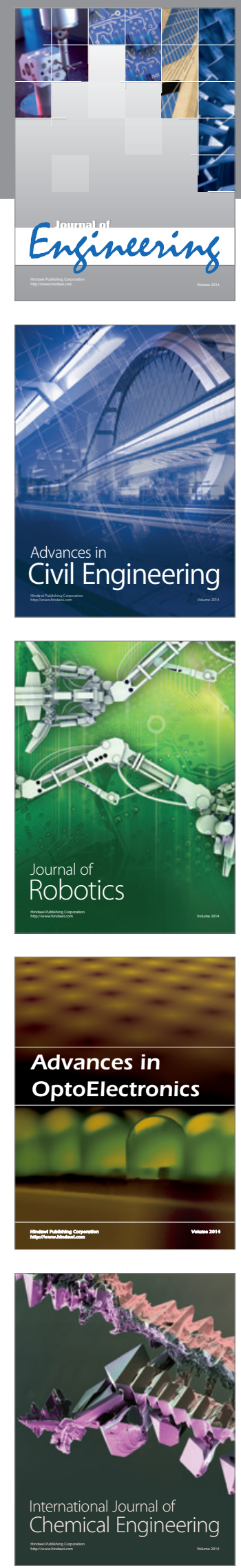

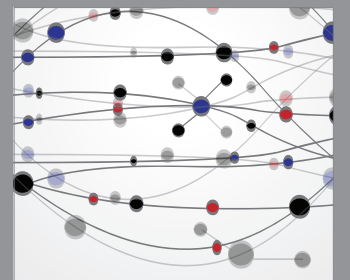

The Scientific World Journal
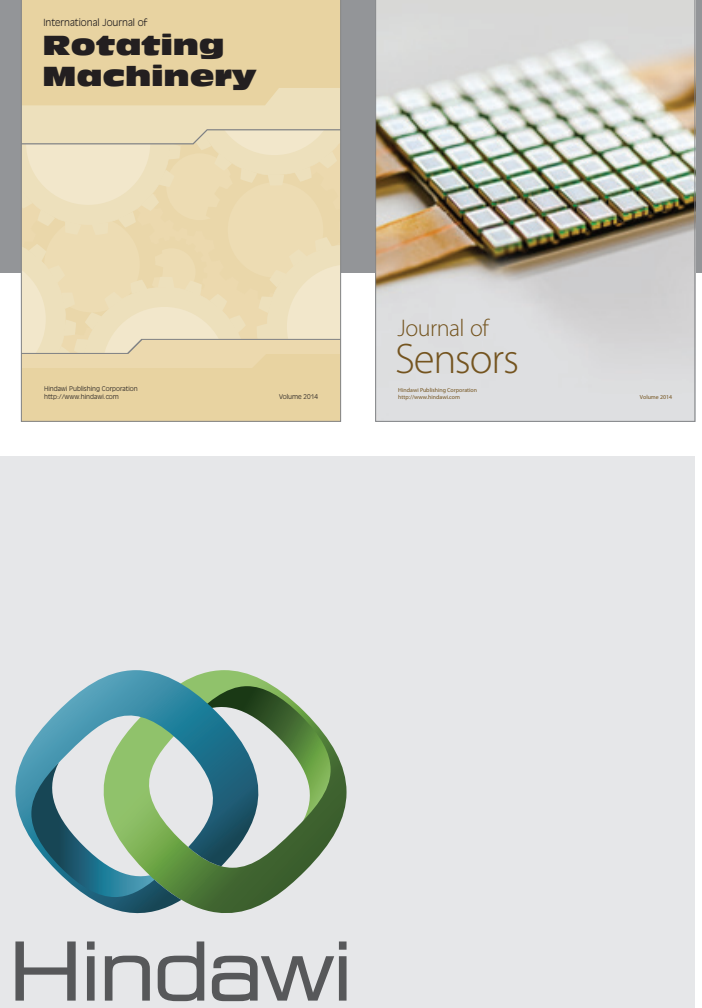

Submit your manuscripts at http://www.hindawi.com
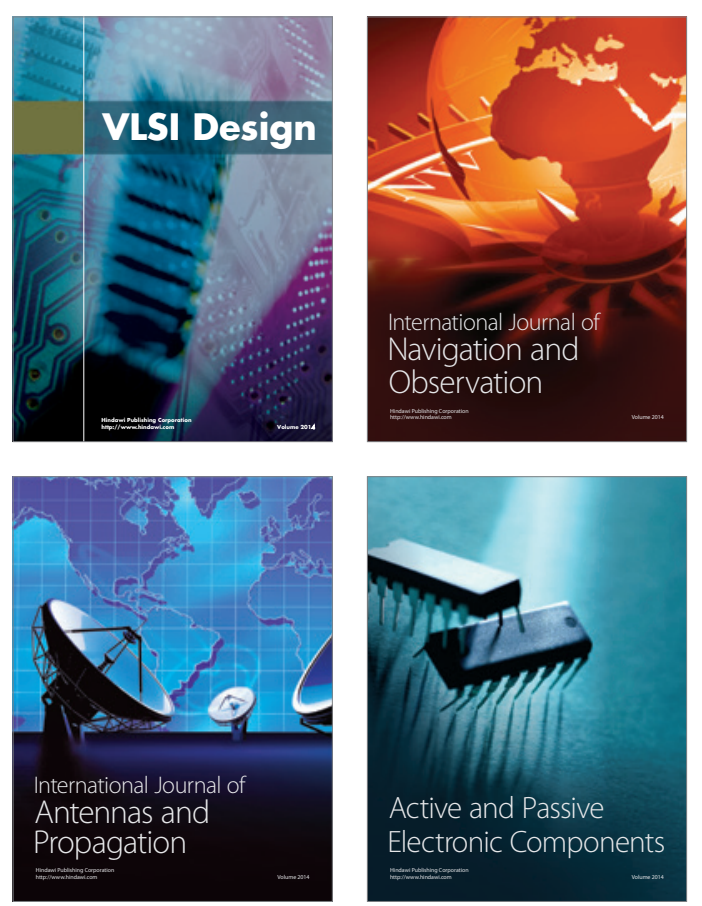
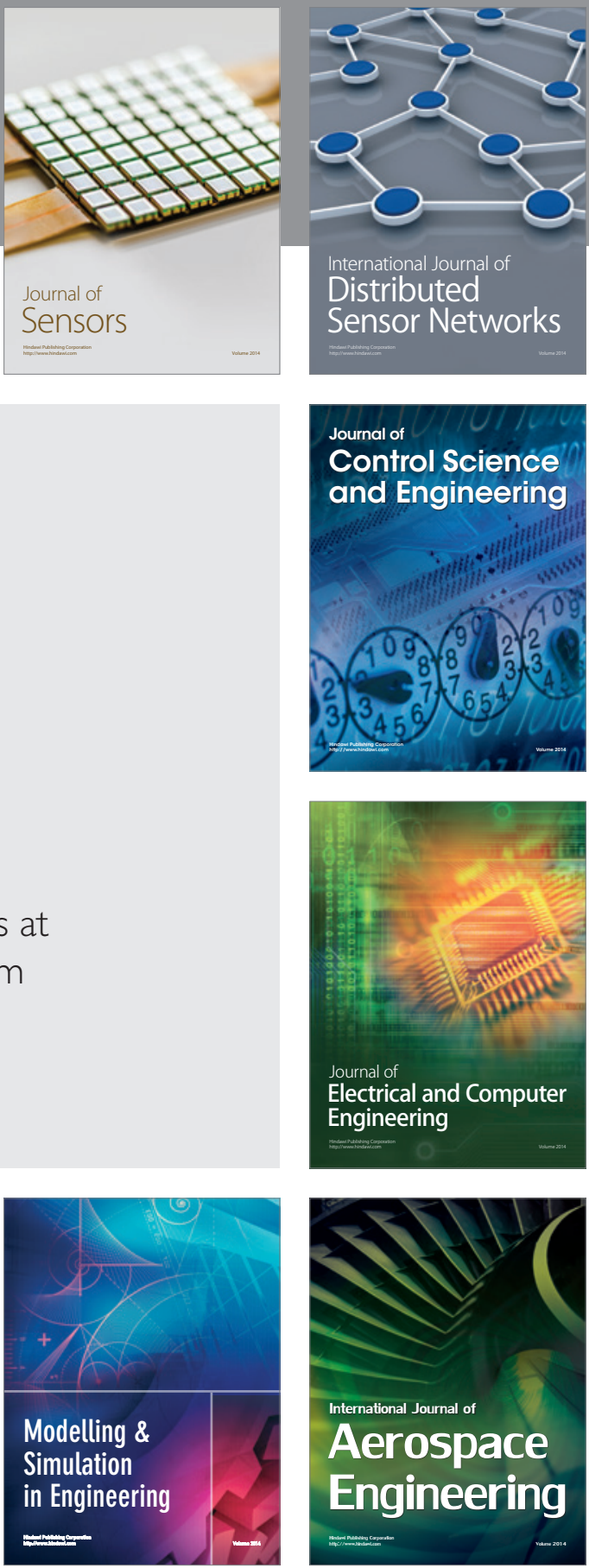

Journal of

Control Science

and Engineering
